\title{
FAST SPECTRAL GALERKIN METHOD FOR LOGARITHMIC SINGULAR EQUATIONS ON A SEGMENT*
}

\author{
Carlos Jerez-Hanckes \\ School of Engineering, Pontificia Universidad Católica de Chile, 782-0436 Santiago, Chile. \\ Email: cjerez@ing.puc.cl \\ Serge Nicaise \\ LAMAV, Université de Valenciennes and CNRS, 59313 Valenciennes, France. \\ Email: serge.nicaise@univ-valenciennes.fr \\ Carolina Urzúa-Torres \\ Seminar for Applied Mathematics, ETH Zurich, Raemistrasse 101, 8092 Zurich, Switzerland. \\ Email: carolina.urzua@sam.math.ethz.ch
}

\begin{abstract}
We present a fast Galerkin spectral method to solve logarithmic singular equations on segments. The proposed method uses weighted first-kind Chebyshev polynomials. Convergence rates of several orders are obtained for fractional Sobolev spaces $\widetilde{H}^{-1 / 2}$ (or $H_{00}^{-1 / 2}$ ). Main tools are the approximation properties of the discretization basis, the construction of a suitable Hilbert scale for weighted $L^{2}$-spaces and local regularity estimates. Numerical experiments are provided to validate our claims.
\end{abstract}

Mathematics subject classification: 65R20, 65F35, 65N22, 65N38.

Key words: Screen problems, Boundary integral operators, Spectral methods.

\section{Introduction}

We study elliptic problems in $\mathbb{R}^{2}$ having as common ground unbounded domains with cuts. Such domains are not even Lipschitz and usually fall in the category of screen, crack or interface problems $[8,27,28,33]$. Our focus lies on the analysis of integral logarithmic singular operators appearing in the associated integral representations. In the simplest scenario, let $\Gamma_{c} \subset \mathbb{R}^{2}$ be an open Jordan curve, $\Omega:=\mathbb{R}^{2} \backslash \bar{\Gamma}_{c}$ an isotropic medium, and consider the following problem: find $u$ such that

$$
\left\{\begin{array}{lll}
-\Delta u=0 & \text { for } & \mathbf{x} \in \Omega \\
u=g & \text { for } & \mathbf{x} \in \Gamma_{c}
\end{array}\right.
$$

where $g$ is a given datum in a suitable functional space. It is well known [22], that the potential $u$ can be represented as the single layer potential:

$$
u(\mathbf{x})=\int_{\Gamma_{c}} \log \frac{1}{\|\mathbf{x}-\mathbf{y}\|} \varphi(\mathbf{y}) \mathrm{d} \mathbf{y}, \quad \text { for } \quad \mathbf{x} \in \Omega,
$$

a representation known as the indirect method [30], wherein $\varphi$ is the solution of the logarithmic singular integral equation:

$$
g(\mathbf{x})=\int_{\Gamma_{c}} \log \frac{1}{\|\mathbf{x}-\mathbf{y}\|} \varphi(\mathbf{y}) \mathrm{d} \mathbf{y}, \quad \text { for } \quad \mathbf{x} \in \Gamma_{c} .
$$

\footnotetext{
* Received January 4, 2016 / Accepted December 5, 2016 /

Published online October 11, 2017 /
} 
This type of first-kind Fredholm equation has received considerable attention in the past $[2,10$, $14,19,31]$ with several strategies put forward to solve it numerically.

More recently, explicit expressions for the inverse of the weakly singular and hypersingular operators over a straight segment $(-1,1)$ as well as Calderón-type identities were provided in [17]. These results were computationally implemented and numerically analyzed for nonuniform low-order discretization in [16]. Still, the number of degrees of freedom required to attain high accuracy remains impractical when performing large scale/numbers of simulations. For example, this is the case when the Monte Carlo method is used to compute statistical moments of the screen problem solutions subject to uncertainties in either geometrical features or sources. As an alternative remedy, one can use $p$-refinement schemes, sometimes referred to as spectral methods $[3,4,7]$. In [13], the authors provide convergence results for polygonal segments. Along both lines of work, Lintner and Bruno [5,6] developed a generalized Calderón formula for open arcs. When combined with their high-order Nyström methods, they observe excellent performance of their Calderón preconditioner for a wide range of geometries and wave propagation problems. However, no mathematical analysis of their method is available.

In this work, we provide a fast spectral Galerkin method to solve general logarithmic singular kernels. Although not a new idea for structures of co-dimension one in $\mathbb{R}^{2}[14,31]$ in the setting of weighted Sobolev spaces, the generalization to classic Sobolev spaces and interface problems seems rather original. Without loss of generality, we will remit to $\Gamma_{c}$ given by bounded Jordan arcs, the case of semi-infinite or infinite cuts not being considered. By changing coordinates, the integral equation is cast over the canonical interval $(-1,1)$, so that it has the form of a compactly perturbed logarithmic integral operator. As we will show, solutions are characterized by squareroot singularities at the endpoints $\{ \pm 1\}$ and weighted Chebyshev polynomials naturally define a basis for numerical approximation. Though we had a different aim, we obtain similar results to those published by Dominguez et al. [15] wherein so-called Hilbert scales are studied along with their connection to weighted Sobolev spaces.

The remaining of this work is organized as follows. In Section 2, we recall certain definitions and introduce notation conventions. Section 2.6 recalls uniqueness and existence results [17] for the exterior Dirichlet problem for the Laplacian in $\mathbb{R}^{2}$ with a line segment removed that lead to (1.3). After presenting Sobolev spaces over $[0, \pi]$ and weighted $L^{2}$-spaces in Sections 3 and 4 , we establish convergence results and truncation errors for associated series expansions in Section 5. Although many of the presented results are well known $[10,21,23]$, we will emphasize the link between trigonometric and Chebyshev polynomials as well as their profound connection with logarithmic operators. In this sense, we extend previous results obtained in the setting of Hölder continuous functions developed in [24,25]. Numerical results are discussed in Section 6 and conclusions drawn in Section 7.

\section{Preliminaries}

\subsection{Model geometry}

We start by recalling the canonic splitting of $\mathbb{R}^{2}$ into two half-planes:

$$
\pi_{ \pm}:=\left\{\mathbf{x}=(x, y) \in \mathbb{R}^{2}: y \lessgtr 0, \forall y \in \mathbb{R}\right\},
$$

with interface $\Gamma$ given by the line $y=0$. The interface is further divided into the open disjoint segments $\Gamma_{c}:=\left(a_{-}, a_{+}\right) \times\{0\}$, where $a_{-}, a_{+} \in \mathbb{R}$ are such that $-\infty<a_{-}<a_{+}<\infty$, and 
$\Gamma_{f}:=\Gamma \backslash \bar{\Gamma}_{c}$. Consequently, the domain over which the partial differential equations will be imposed is $\Omega:=\mathbb{R}^{2} \backslash \bar{\Gamma}_{c}$. Furthermore, we define the canonic segment $\hat{\Gamma}_{c}:=(-1,1) \times\{0\}$ and connect it to the interval $I:=[0, \pi]$ via the mapping $x \mapsto \cos \theta$ when taking its closure.

Later on we will assume $\Gamma$ to be a Jordan curve, i.e. there exists a bijective parametrization $\vartheta: \mathbb{R} \rightarrow \mathbb{R}^{2}$ such that $\Gamma=\vartheta(\mathbb{R})$, with the consequent splitting of $\mathbb{R}^{2}$,

$$
\pi_{ \pm}:=\left\{\mathbf{x} \in \mathbb{R}^{2}: y \lessgtr \vartheta(t), \forall t \in \mathbb{R}\right\},
$$

and define $\Gamma_{c}$ as a bounded segment in $\Gamma$.

\subsection{Notation}

Let $D \subseteq \mathbb{R}^{d}$, with $d=1,2$, be a Lipschitz bounded or unbounded domain. We denote by $\mathcal{C}^{k}(D)$ the space of $k$-times differentiable continuous functions over $D$ with $k \in \mathbb{N}_{0}$, with $\mathbb{N}_{0}:=\mathbb{N} \cup\{0\}$. Its subspace of compactly supported functions is $\mathcal{C}_{0}^{k}(D)$ and for infinitely differentiable functions we write $\mathcal{D}(D) \equiv \mathcal{C}_{0}^{\infty}(D)$. The space of distributions or linear functionals over $\mathcal{D}(D)$ is $\mathcal{D}^{\prime}(D)$. Also, let $L^{p}(D)$ be the standard equivalence class of functions with bounded $L^{p}$-norm over $D$. Duality products are denoted by angular brackets, $\langle\cdot, \cdot\rangle$, with subscripts accounting for the duality pairing by stating only the functional space of the second argument. Inner products are denoted by round brackets, $(\cdot, \cdot)$, with integration domains or functional spaces specified by subscripts. Furthermore, operators are denoted in mild calligraphic style with the exception of $\mathrm{D}(c f$. (4.18)) - and complex conjugates by overline. Finally in the whole paper, the notation $a \lesssim b$ is used for the estimate $a \leq C b$, where $C$ is a generic positive constant that does not depend on $a, b$ nor on any polynomial degree $N$.

\subsection{Standard Sobolev spaces}

For $s \in \mathbb{R}, H^{s}\left(\mathbb{R}^{d}\right)$ are the classic Sobolev spaces $[1,32]$ with $H^{0}\left(\mathbb{R}^{d}\right) \equiv L^{2}\left(\mathbb{R}^{d}\right)$. Let $D \subseteq \mathbb{R}^{d}$ be a non-empty open set, one writes

$$
H^{s}(D)=\left\{u \in \mathcal{D}^{\prime}(D): u=\left.U\right|_{D} \quad \text { for some } U \in H^{s}\left(\mathbb{R}^{d}\right)\right\} .
$$

For $s \geq 0$, we say that a distribution belongs to the local Sobolev space $H_{\mathrm{loc}}^{s}\left(\mathbb{R}^{d}\right)$ if its restriction to every compact set $K \Subset \mathbb{R}^{d}$ lies in $H^{s}(K)$. If $D$ has a boundary, we assume that it can be extended over a closed manifold $\widetilde{D}$ and write $\widetilde{u}$ for the extension of $u$ by zero over $\widetilde{D} \backslash D$. For $s>0$ and $D$ Lipschitz, it holds

$$
\widetilde{H}^{s}(D):=\left\{u \in H^{s}(D): \widetilde{u} \in H^{s}(\widetilde{D})\right\},
$$

provided with the norm $\|u\|_{\widetilde{H}^{s}(D)}=\|\widetilde{u}\|_{H^{s}(\widetilde{D})}$. If $D$ is a bounded domain in $\mathbb{R}^{d}$ then one uses $\widetilde{D}:=\mathbb{R}^{d}$ and if $D$ is closed $\widetilde{H}^{s}(D) \equiv H^{s}(D)$. For negative $s$, we identify $\widetilde{H}^{s}(D)$ with the dual space of $H^{-s}(D)$. In particular,

$$
\widetilde{H}^{-1 / 2}(D) \equiv\left(H^{1 / 2}(D)\right)^{\prime} \quad \text { and } \quad H^{-1 / 2}(D) \equiv\left(\widetilde{H}^{1 / 2}(D)\right)^{\prime} .
$$

If $D$ is Lipschitz and bounded, let $\varrho:=\operatorname{dist}(\mathbf{x}, \partial D)$ be the Euclidean distance from $\mathbf{x}$ to $\partial D$. With this, we recall the following result $[12,20]$ : 
Lemma 2.1. Let $0 \leq s<\frac{1}{2}$. Then, the application

$$
u \longmapsto \varrho^{-s} u
$$

is linear and continuous from $H^{s}(D)$ to $L^{2}(D)$. Moreover, if $u \in \widetilde{H}^{1 / 2}(D)$ and $\partial D$ Lipschitz, then $\widetilde{u} \in H^{1 / 2}\left(\mathbb{R}^{d}\right)$ and

$$
\varrho^{-1 / 2} u \in L^{2}(D)
$$

are equivalent characterizations.

\subsection{Traces}

Define restrictions $u^{ \pm}:=\left.u\right|_{\pi_{ \pm}}$. As customary, we introduce the interior trace operators $\gamma^{ \pm}: \mathcal{D}\left(\bar{\pi}_{ \pm}\right) \rightarrow \mathcal{D}(\Gamma)$ as

$$
\gamma^{ \pm} u:=\lim _{\epsilon \rightarrow 0^{ \pm}} u(x, \epsilon)=\gamma^{ \pm} u^{ \pm} .
$$

If $s>\frac{1}{2}$, the operators $\gamma^{ \pm}$have unique extensions to bounded linear operators $H_{\mathrm{loc}}^{s}\left(\pi_{ \pm}\right) \rightarrow$ $H_{\mathrm{loc}}^{s-1 / 2}(\Gamma)$. We will denote by $\gamma_{c}^{ \pm}$the restriction of the trace operator to a bounded $\Gamma_{c}$. If $s>\frac{1}{2}$, a unique extension to a bounded linear operator $\gamma_{c}^{ \pm}: H_{\mathrm{loc}}^{s}\left(\pi_{ \pm}\right) \rightarrow H^{s-1 / 2}\left(\Gamma_{c}\right)$ can be obtained by density of $\mathcal{D}\left(\bar{\pi}_{ \pm}\right)$in $H^{s}\left(\pi_{ \pm}\right)$.

The symbol $[\gamma]:=\gamma^{+}-\gamma^{-}$represents the jump operator across $\Gamma$. Normal derivatives are written $\frac{\partial}{\partial n}=\mathbf{n} \cdot \nabla$ with $\mathbf{n}$ pointing outwards for closed boundaries. In the case of $\Gamma$, being a non-orientable manifold of codimension one, we assume $\mathbf{n}$ pointing along the positive $y$-axis.

\subsection{Weighted Sobolev spaces}

Since the problem domain is unbounded, one usually works in either local Sobolev spaces or in the following weighted Sobolev space [26] :

$$
W^{1,-1}(\Omega)=\left\{u \in \mathcal{D}^{\prime}(\Omega): \frac{u}{\left(1+r^{2}\right)^{1 / 2} \log \left(2+r^{2}\right)} \in L^{2}(\Omega), \nabla u \in L^{2}(\Omega)\right\},
$$

where $r:=\|\mathbf{x}\|$ with $\mathbf{x} \in \mathbb{R}^{2}$. This space coincides with the standard $H_{\text {loc }}^{1}(\Omega)$ for a bounded part of $\Omega$, which avoids specifying decaying behaviors at infinity [26]. Furthermore, this weighted space is a Hilbert one, whereas local Sobolev spaces are only of Fréchet-type.

Now, traces along $\Gamma$ for elements in $W^{1,-1}(\Omega)$ lie in the usual $H_{\text {loc }}^{1 / 2}(\Gamma)$, and their restriction to a bounded $\Gamma_{c}$ generates the subspace $H^{1 / 2}\left(\Gamma_{c}\right)$. Lastly, let us introduce two more spaces: $\widetilde{H}_{\langle 0\rangle}^{-1 / 2}\left(\Gamma_{c}\right)$ as the subspace of $\widetilde{H}^{-1 / 2}\left(\Gamma_{c}\right)$-distributions with zero mean value, i.e.

$$
\widetilde{H}_{\langle 0\rangle}^{-1 / 2}\left(\Gamma_{c}\right)=\left\{\varphi \in \widetilde{H}^{-1 / 2}\left(\Gamma_{c}\right):\langle\varphi, 1\rangle_{H^{1 / 2}\left(\Gamma_{c}\right)}=0\right\},
$$

and $H_{*}^{1 / 2}\left(\Gamma_{c}\right)$ as the space of functions in $H^{1 / 2}\left(\Gamma_{c}\right)$ satisfying

$$
\int_{a_{-}}^{a_{+}} \frac{1}{\sqrt{\left(a_{+}-t\right)\left(t-a_{-}\right)}} g(t) d t=0 .
$$




\subsection{Laplace problem with Dirichlet boundary condition}

We start by considering the Laplace problem with Dirichlet conditions over a bounded segment $\Gamma_{c}$. Let $g \in H^{1 / 2}\left(\Gamma_{c}\right)$, we seek $u \in W^{1,-1}(\Omega)$ such that:

$$
\begin{cases}-\Delta u=0 & \text { on } \Omega \\ \gamma_{c}^{ \pm} u=g & \text { over } \Gamma_{c} .\end{cases}
$$

Proposition 2.1 (Prop. 2.7 and Cor. 2.9 in [17]) If $g \in H^{1 / 2}\left(\Gamma_{c}\right)$, then (2.11) has a unique solution in $W^{1,-1}(\Omega)$ whose Neumann jump at $\Gamma_{c}$ belongs to the space $\widetilde{H}_{\langle 0\rangle}^{-1 / 2}\left(\Gamma_{c}\right)$.

We return to our original integral equation (1.3), now over $\hat{\Gamma}_{c}$ :

$$
\mathcal{L}[\varphi](\mathbf{x}):=\int_{\hat{\Gamma}_{c}} \log \frac{1}{\|\mathbf{x}-\mathbf{y}\|} \varphi(\mathbf{y}) \mathrm{d} \mathbf{y}=g(\mathbf{x}), \quad \forall \mathbf{x} \in \hat{\Gamma}_{c},
$$

and recall the following proposition.

Proposition 2.2 (Prop. 3.1 in [17]) The integral equation (2.12) admits a variational formulation in the Hilbert space $\widetilde{H}_{\langle 0\rangle}^{-1 / 2}\left(\hat{\Gamma}_{c}\right)$ equal to

$$
\left\langle\mathcal{L} \varphi, \varphi^{t}\right\rangle_{\widetilde{H}^{-1 / 2}\left(\hat{\Gamma}_{c}\right)}=\left\langle g, \varphi^{t}\right\rangle_{\widetilde{H}^{-1 / 2}\left(\hat{\Gamma}_{c}\right)}, \quad \forall \varphi^{t} \in \widetilde{H}_{\langle 0\rangle}^{-1 / 2}\left(\hat{\Gamma}_{c}\right) .
$$

$\mathcal{L}$ is a bijection between $\widetilde{H}_{\langle 0\rangle}^{-1 / 2}\left(\hat{\Gamma}_{c}\right)$ and the subspace $H_{*}^{1 / 2}\left(\hat{\Gamma}_{c}\right)$ and the associated bilinear form is coercive, i.e.

$$
\langle\mathcal{L} \varphi, \varphi\rangle_{\widetilde{H}^{-1 / 2}\left(\hat{\Gamma}_{c}\right)} \gtrsim\|\varphi\|_{\widetilde{H}^{-1 / 2}\left(\hat{\Gamma}_{c}\right)}^{2}, \quad \forall \varphi \in \widetilde{H}_{\langle 0\rangle}^{-1 / 2}\left(\hat{\Gamma}_{c}\right) .
$$

The above variational formulation constitutes the main equation to be solved. Key to the proposed numerical scheme is the analysis of the singular behavior of Neumann jumps at the segment endpoints.

\subsection{Local regularity results.}

Let $\left(r_{ \pm}, \theta_{ \pm}\right)$denote local coordinates in $\Omega$ centered at the endpoints $a_{ \pm}$of $\Gamma_{c}$, respectively, and such that $\theta_{ \pm}=0$ when on $\Gamma_{c}$ from above and $\theta_{ \pm}=2 \pi$ from below. Fix cut-off functions $\eta_{ \pm}$such that $\eta_{ \pm} \equiv 1$ near the corresponding endpoints and $\eta_{ \pm} \equiv 0$ near the other endpoint. If $g$ is sufficiently smooth in (2.11), the potential satisfies ( $c f .[12])$ :

$$
\eta_{ \pm} u=c_{1 \pm} \eta_{ \pm} r_{ \pm}^{1 / 2} \sin \frac{\theta_{ \pm}}{2}+c_{2 \pm} \eta_{ \pm} r_{ \pm}^{3 / 2} \sin \frac{3 \theta_{ \pm}}{2}+u_{R \pm},
$$

with $u_{R \pm} \in H_{\mathrm{loc}}^{s}(\Omega)$ and $s$ such that $\frac{3}{2}<s-1<\frac{5}{2}$. The coefficients $c_{i}, i=1,2$, are real numbers. Let $\rho(x):=\operatorname{dist}\left(x, a_{-}\right) \operatorname{dist}\left(x, a_{+}\right)=\left|x-a_{-}\right|\left|x-a_{+}\right|$, represent a sort of distance from any point in $\Gamma_{c}$ to the closest endpoint in $\partial \Gamma_{c}$. Thus, in local polar coordinates -by taking $\theta_{ \pm}=\{0,2 \pi\}$ and using the equivalence between $\rho$ and $r_{-}$, the above decomposition implies that for the Neumann jump $\varphi \in \widetilde{H}_{\langle 0\rangle}^{-1 / 2}\left(\Gamma_{c}\right)$, it holds

$$
\eta_{ \pm} \varphi=\eta_{ \pm}\left[\frac{\partial u}{\partial n}\right]=c_{1 \pm}^{\prime} \eta_{ \pm} \rho^{-1 / 2}+c_{2 \pm}^{\prime} \eta_{ \pm} \rho^{1 / 2}+\varphi_{R \pm},
$$


where $\varphi_{R \pm} \in \widetilde{H}^{s-3 / 2}(\Gamma)$ for $\frac{5}{2}<s<\frac{7}{2}$ and the coefficients $c_{i}^{\prime}, i=1,2$, are still real numbers. This decomposition implies that

$$
\varphi=c_{1}^{\prime} \rho^{-1 / 2}+c_{2}^{\prime} \rho^{1 / 2}+\varphi_{R}+\psi
$$

where $\varphi_{R}:=\varphi_{R+}+\varphi_{R-}+\left(1-\eta_{+}-\eta_{-}\right) \varphi \in \widetilde{H}^{s-3 / 2}(\Gamma)$ for $\frac{5}{2}<s<\frac{7}{2}, c_{i}^{\prime}:=c_{i+}^{\prime}+c_{i-}^{\prime}$ and

$$
\psi:=\rho^{-1 / 2} \sum_{ \pm}\left(c_{1 \pm}^{\prime}\left(\eta_{ \pm}-1\right)+c_{2 \pm}^{\prime}\left(\eta_{ \pm}-1\right) \rho\right) .
$$

\section{Trigonometric Bases and Sobolev Spaces in $[0, \pi]$}

Let us consider the following spectral value Sturm-Liouville problem with Neumann conditions over $I:=[0, \pi]$. More precisely, we look for $\lambda \in \mathbb{C}$ and $u \neq 0$ in $\mathcal{C}^{1}(I)$, dependent on $\lambda$, such that

$$
\begin{cases}-u^{\prime \prime}=\lambda u & \text { in } I, \\ u^{\prime}=0 & \text { on } \partial I .\end{cases}
$$

Solutions clearly take the form:

$$
u(\theta)=A \cos (\sqrt{\lambda} \theta)+B \sin (\sqrt{\lambda} \theta),
$$

with derivative

$$
u^{\prime}(\theta)=-\sqrt{\lambda} A \sin (\sqrt{\lambda} \theta)+\sqrt{\lambda} B \cos (\sqrt{\lambda} \theta) .
$$

Upon imposing boundary conditions, we obtain $B=0$, set $A=1$ and retrieve the set of eigenpairs $\left(\lambda_{m}, u_{m}\right)=\left(m^{2}, \cos m \theta\right)$ for $m \in \mathbb{Z}$. Since for negative and positive $m$ the associated eigenpairs are identical, it holds

Proposition 3.1 ([23]) The set of trigonometric polynomials:

$$
e_{m}^{\cos }(\theta):= \begin{cases}1 / \sqrt{\pi} & \text { for } m=0, \\ \sqrt{\frac{2}{\pi}} \cos m \theta & \text { for } m \geq 1,\end{cases}
$$

with $m \in \mathbb{N}_{0}$, constitutes an orthonormal basis for $L^{2}(I)$.

As a consequence of Proposition 3.1, we can write any function $\varphi$ in $L^{2}(I)$ as a series expansion:

$$
\varphi(\theta)=\sum_{m \in \mathbb{N}_{0}} a_{m}^{\cos } e_{m}^{\cos }(\theta),
$$

where the complex coefficients $a_{m}^{\text {cos }}$ are those of the cosine-Fourier transform of $\varphi$ :

$$
a_{m}^{\cos }:=\int_{0}^{\pi} \varphi(\theta) e_{m}^{\cos }(\theta) d \theta .
$$

If $s \in[0, \infty)$, we denote by $H_{\cos }^{s}(I)$ the Sobolev space of functions $\varphi$ in $L^{2}(I)$ with the property

$$
\sum_{m \in \mathbb{N}_{0}} \underline{m}^{2 s}\left|a_{m}^{\cos }\right|^{2}<\infty
$$


for the cosine Fourier coefficients $a_{m}^{\cos }$ of $\varphi$ with

$$
\underline{m}:=\left\{\begin{array}{lll}
1 & \text { for } & m=0 \\
m & \text { for } & m \in \mathbb{N}
\end{array} .\right.
$$

Notice that $H_{\mathrm{cos}}^{0}(I)$ coincides with $L^{2}(I)$.

Theorem $3.1([29])$ The Sobolev space $H_{\mathrm{cos}}^{s}(I)$ is a Hilbert space with the inner product defined by

$$
(\varphi, \psi)_{H_{\cos }^{s}(I)}:=\sum_{m \in \mathbb{N}_{0}} \underline{m}^{2 s} a_{m}^{\cos } \overline{b_{m}^{\cos }}
$$

for $\varphi, \psi \in H_{\cos }^{s}(I)$ with coefficients $a_{m}^{\cos }$ and $b_{m}^{\cos }$, respectively. Consequently, the norm on $H_{\mathrm{cos}}^{s}(I)$ is given by

$$
\|\varphi\|_{H_{\text {cos }}^{s}(I)}^{2}=\sum_{m \in \mathbb{N}_{0}} \underline{m}^{2 s}\left|a_{m}^{\cos }\right|^{2} .
$$

Notice that the above definition extends to complex-valued elements.

Remark 3.1. Alternatively, for $k \in \mathbb{N}$, we can make the identification:

$$
H_{\mathrm{cos}}^{k}(I) \equiv \operatorname{dom}\left(\Delta_{N}^{k / 2}\right),
$$

where $\operatorname{dom}\left(\Delta_{N}\right)$ is the domain of the Laplacian with zero Neumann conditions over $I$ :

$$
\operatorname{dom}\left(\Delta_{N}\right)=\left\{\varphi \in H^{2}(I): \varphi^{\prime}(0)=\varphi^{\prime}(\pi)=0\right\},
$$

and $\operatorname{dom}\left(\Delta_{N}^{1 / 2}\right)=H^{1}(I)$. For example, $H_{\cos }^{4}(I)=\operatorname{dom}\left(\Delta_{N}^{2}\right)$ is given by

$$
\begin{aligned}
\operatorname{dom}\left(\Delta_{N}^{2}\right) & =\left\{\varphi \in \operatorname{dom}\left(\Delta_{N}\right): \Delta \varphi \in \operatorname{dom}\left(\Delta_{N}\right)\right\} \\
& =\left\{\varphi, \varphi^{(2)} \in H^{2}(I) \varphi^{(1)}(0)=\varphi^{(1)}(\pi)=0, \varphi^{(3)}(0)=\varphi^{(3)}(\pi)=0\right\} .
\end{aligned}
$$

Now, by injection of Sobolev spaces into continuous ones [1], it holds for $k \geq 2$ :

$$
H_{\mathrm{cos}}^{k}(I)=\left\{\varphi \in H^{k}(I): \varphi^{(2 j+1)}(0)=\varphi^{(2 j+1)}(\pi)=0, \forall j=0, \ldots,\left\lfloor\frac{k}{2}-1\right\rfloor\right\},
$$

where $\lfloor\cdot\rfloor$ denotes the largest integer less or equal than the argument.

We are interested in finding the best approximation over the above defined Sobolev spaces. For $N \geq 0$, let $\Pi_{N}^{\cos }(I)$ denote the $(N+1)$-dimensional space spanned by the cosine basis up to degree $N$ in $[0, \pi]$. Let $\mathcal{P}_{N}^{\cos }: H_{\cos }^{s}(I) \longrightarrow \Pi_{N}^{\cos }(I)$ be the orthogonal projection in the inner product of $L^{2}(I)$, i.e. if $\varphi \in H_{\cos }^{s}(I)$, for $s \geq 0$, it holds

$$
\left(\varphi-\mathcal{P}_{N}^{\cos } \varphi, \phi\right)_{L^{2}(I)}=0, \quad \forall \phi \in \Pi_{N}^{\cos }(I),
$$

and which approximates $\varphi$ by the finite sum:

$$
\mathcal{P}_{N}^{\cos }[\varphi](x)=\sum_{m=0}^{N} a_{m}^{\cos } e_{m}^{\cos }(x) .
$$

Moreover, $\mathcal{P}_{N}^{\cos } \varphi$ is the best approximation in the $L^{2}$-norm among all functions in $\Pi_{N}^{\cos }(I)$. 
Theorem 3.2. Let $r, s \in \mathbb{R}$ with $0 \leq s \leq r$ and let $N$ be a positive integer. Then, the following projection error holds

$$
\left\|\varphi-\mathcal{P}_{N}^{\cos } \varphi\right\|_{H_{\mathrm{cos}}^{s}(I)} \leq N^{s-r}\|\varphi\|_{H_{\mathrm{cos}}^{r}(I)}
$$

for $\varphi \in H_{\mathrm{cos}}^{r}(I)$.

Proof. One can write

$$
\begin{aligned}
\left\|\varphi-\mathcal{P}_{N}^{\cos } \varphi\right\|_{H_{\mathrm{cos}}^{s}(I)}^{2} & =\sum_{m>N} \underline{m}^{2 s-2 r+2 r}\left|a_{m}^{\cos }\right|^{2} \\
& \leq N^{2(s-r)} \sum_{m>N} \underline{m}^{2 r}\left|a_{m}^{\cos }\right|^{2} \leq N^{2(s-r)}\|\varphi\|_{H_{\cos }^{r}(I)}^{2},
\end{aligned}
$$

and the statement is attained after taking square roots.

Remark 3.2. This result shows that the more regular $u$ is, the better the approximation by $\mathcal{P}_{N}^{\cos } \varphi$. More precisely, if we take the $L^{2}$-norm, i.e. for $s=0$, the error is of order $\mathcal{O}\left(N^{-r}\right)$ and if $\varphi$ is analytic one achieves exponential or spectral convergence, i.e. $\mathcal{O}\left(e^{-\eta N}\right)[7,34]$.

\section{Weighted $L^{2}$-spaces and Chebyshev Polynomials}

The Chebyshev polynomials $T_{n}(x)$ and $U_{n}(x)$ of the first and second kinds, respectively, are polynomials of degree $n$, defined in $x \in[-1,1]$ as:

$$
T_{n}(x)=\cos n \theta \quad \text { and } \quad U_{n}(x)=\frac{\sin (n+1) \theta}{\sin \theta}
$$

with $x=\cos \theta, \theta \in[0, \pi]$. These satisfy the recurrence relations:

$$
P_{n}(x)=2 x P_{n-1}(x)-P_{n-2}(x), \quad n=2,3, \ldots,
$$

together with initial conditions $T_{0}(x)=1, T_{1}(x)=x, U_{0}(x)=1$ and $U_{1}(x)=2 x$. Furthermore, it holds

$$
\begin{aligned}
& U_{n}(x)-U_{n-1}(x)=2 T_{n}(x), \\
& T_{n}^{\prime}(x)=n U_{n-1}(x) \\
& \left(w U_{n-1}\right)^{\prime}(x)=-n w^{-1}(x) T_{n}(x),
\end{aligned}
$$

for $n \in \mathbb{N}$, where the weight function $w$ is given by

$$
w(x):=\sqrt{1-x^{2}} \quad \text { for } x \in[-1,1] .
$$

Moreover, the $T_{n}$ are orthogonal with respect to $w^{-1}$ :

$$
\int_{-1}^{1} T_{n}(x) T_{m}(x) w^{-1}(x) d x= \begin{cases}0 & n \neq m \\ \pi / 2 & n=m \neq 0 \\ \pi & n=m=0 .\end{cases}
$$

For the second kind Chebyshev polynomials $U_{n}$, it holds

$$
\int_{-1}^{1} U_{n}(x) U_{m}(x) w(x) d x= \begin{cases}0 & n \neq m \\ \pi / 2 & n=m \neq 0 .\end{cases}
$$


Based on the above, we can define the weighted function spaces and norms:

$$
\begin{aligned}
& L_{1 / w}^{2}:=\left\{u \text { measurable }:\|f\|_{1 / w}^{2}:=\int_{-1}^{1}|f(x)|^{2} w^{-1}(x) d x<\infty\right\}, \\
& L_{w}^{2}:=\left\{u \text { measurable }:\|f\|_{w}^{2}:=\int_{-1}^{1}|f(x)|^{2} w(x) d x<\infty\right\},
\end{aligned}
$$

and the associated space:

$$
W=\left\{u \text { measurable : } u \in L_{1 / w}^{2}, u^{\prime} \in L_{w}^{2}\right\},
$$

with graph norm

$$
\|u\|_{W}=\|u\|_{1 / w}+\left\|u^{\prime}\right\|_{w} .
$$

Proposition 4.1. There is a continuous inclusion $L_{1 / w}^{2} \subset L_{w}^{2}$. Define $\mathcal{W}$ and $\mathcal{W}^{-1}$ by $\mathcal{W} \varphi:=$ $w \varphi$ and $\mathcal{W}^{-1} \varphi=w^{-1} \varphi$, then they are isometries, namely

$$
\mathcal{W}: L_{w}^{2} \rightarrow L_{1 / w}^{2} \quad \text { and } \quad \mathcal{W}^{-1}: L_{1 / w}^{2} \rightarrow L_{w}^{2},
$$

are isometries.

Proof. Let $u \in L_{1 / w}^{2}$. Since all terms are positive, we get

$$
\|u\|_{w}^{2}=\int_{-1}^{1}|u(x)|^{2} \frac{w^{2}(x)}{w(x)} d x \leq\left(\int_{-1}^{1} w^{2}(x) d x\right)\|u\|_{1 / w}^{2} \lesssim\|u\|_{1 / w}^{2}
$$

and the inclusion follows. The isometries are found from the norm definitions: if $\varphi \in L_{w}^{2}$,

$$
\|\varphi\|_{w}=\int_{-1}^{1} w(x)|\varphi(x)|^{2} d x=\int_{-1}^{1} \frac{|\psi(x)|^{2}}{w(x)} d x=\|\psi\|_{1 / w},
$$

where $\psi=w \varphi \in L_{1 / w}^{2}$ and, if $\varphi$ is in $L_{1 / w}^{2}$, application of $\mathcal{W}^{-1}$ yields

$$
\|\varphi\|_{1 / w}=\left\|w^{-1} \varphi\right\|_{w}
$$

which concludes the proof.

Lemma 4.1 ([29]) The spaces $L_{w}^{2}, L_{1 / w}^{2}$ and $W$ are Hilbert spaces with inner products inducing the norms $\|\cdot\|_{w},\|\cdot\|_{1 / w}$ and $\|\cdot\|_{W}$ :

$$
\begin{aligned}
& (u, v)_{w^{ \pm 1}}=\int_{-1}^{1} u(t) \bar{v}(t) w^{ \pm 1}(t) d t \\
& (u, v)_{W}=(u, v)_{1 / w}+\left(u^{\prime}, v^{\prime}\right)_{w} .
\end{aligned}
$$

One can generalize the above definitions to spaces of order $k \in \mathbb{N}_{0}$. First, define the following first-order differential operator:

$$
\mathrm{D}:=-w \frac{d}{d x}
$$

and denote by $\mathrm{D}^{n}$ the composition $\mathrm{D} \circ \ldots \circ \mathrm{D} n$-times. With this, we define the weighted spaces as

$$
W^{k}:=\left\{u \text { measurable : } \mathrm{D}^{l} u \in L_{1 / w}^{2}, \quad \forall l=0, \ldots, k\right\}
$$


with norm

$$
\|u\|_{W^{k}}^{2}=\sum_{l=0}^{k}\left\|\mathrm{D}^{l} u\right\|_{1 / w}^{2}
$$

and induced inner product. Clearly, $W^{0}=L_{1 / w}^{2}$ and $W^{1}=W$. Finally, we derive a result that will be used later on.

Proposition 4.2. Let $m \in \mathbb{N}_{0}$. Then, $W^{m}$ is continuously imbedded in $L^{2}((-1,1))$.

Proof. By definition, it holds $W^{m} \hookrightarrow L_{1 / w}^{2}$ for $m \geq 1$ and hence, we only need to prove the above for $L_{1 / w}^{2}$. Let $u \in L_{1 / w}^{2}$ and take the standard $L^{2}$-norm over the interval $(-1,1)$ :

$$
\|u\|_{L^{2}((-1,1))}^{2}=\int_{-1}^{1}|u(x)|^{2} d x=\int_{-1}^{1}|u(x)|^{2} \frac{w(x)}{w(x)} d x \leq\|u\|_{1 / w}^{2},
$$

since the $L^{\infty}$-norm of $w$ is equal to one.

\subsection{Link with $H_{\mathrm{cos}}^{s}(I)$ spaces}

We now use the mapping $x=\cos \theta$ which takes $I \mapsto[-1,1]$, with measure given by $d x=$ $-\sin \theta d \theta$, to define

$$
\check{u}(\theta):=u(\cos \theta),
$$

and present some results concerning the relation between the weighted $L^{2}$-spaces just defined and the Sobolev spaces over the segment $I$ introduced in Section 3.

Proposition 4.3. The spaces $L_{1 / w}^{2}$ and $L^{2}(I)$ are isometric.

Proof. We note that the change of variables $x=\cos \theta$ transforms the space

$$
u \in L_{1 / w}^{2} \longmapsto \check{u} \in L^{2}(I) .
$$

Moreover, the transformation is isometric since

$$
\|u\|_{1 / w}=\int_{-1}^{1} \frac{|u(x)|^{2}}{w(x)} d x=\int_{0}^{\pi}|\check{u}(\theta)|^{2} d \theta=\|\check{u}\|_{L^{2}(I)}
$$

as stated.

Proposition 4.4. An isometric isomorphism between $W$ and $H_{\mathrm{cos}}^{1}(I)$ exists.

Proof. Let $u \in H_{\mathrm{cos}}^{1}(I)$. Then, the derivative can be written as

$$
\check{u}^{\prime}(\theta)=u^{\prime}(\cos \theta)(-\sin \theta)=-u^{\prime}(x) w(x) .
$$

Taking the $L^{2}$-norm gives

$$
\left\|\check{u}^{\prime}\right\|_{L^{2}(I)}^{2}=\int_{-1}^{1}\left|w(x) u^{\prime}(x)\right|^{2} w^{-1}(x) d x=\int_{-1}^{1}\left|w^{1 / 2}(x) u^{\prime}(x)\right|^{2} d x=\left\|u^{\prime}\right\|_{w}^{2}
$$

so that $w^{1 / 2} u^{\prime} \in L^{2}((-1,1))$. By Proposition 4.3 and the definition of $W$, the statement follows.

Now, the question is whether for all non-negative integers $k, \breve{u} \in H_{\cos }^{k}(I)$ implies $u$ in $W^{k}$. The answer is positive: 
Proposition 4.5. Let $k \in \mathbb{N}_{0}$. There is an isometric isomorphism between the spaces $W^{k}$ and $H_{\mathrm{cos}}^{k}(I)$ defined by $(4.22)$.

Proof. We focus on the case $k \geq 2$ as for $k=0,1$ this is already shown in Propositions 4.3 and 4.4. Let $u \in H_{\cos }^{k}(I)$. From the definition of $W^{k}$, we need to bound the derivatives $\mathrm{D}^{l} u$ in the $L_{1 / w}^{2}$-norm for $l=0, \ldots k$, i.e.

$$
\left\|\mathrm{D}^{l} u\right\|_{1 / w}^{2}=\int_{-1}^{1}\left|\mathrm{D}^{l} u(x)\right|^{2} \frac{d x}{w(x)}<\infty
$$

Mapping $x$ into $\cos \theta$ gives

$$
\int_{0}^{\pi}\left|\check{D}^{l} \check{u}(\theta)\right|^{2} d \theta<\infty
$$

On the other hand, it holds

$$
\check{\mathrm{D}}=-\sin \theta\left(\frac{d \theta}{d x} \frac{d}{d \theta}\right)=\frac{d}{d \theta},
$$

and so $\mathrm{D}^{l} u$ is mapped into $\check{u}^{(l)}$. Consequently,

$$
\int_{0}^{\pi}\left|\check{D}^{l} \check{u}(\theta)\right|^{2} d \theta=\int_{0}^{\pi}\left|\check{u}^{(l)}(\theta)\right|^{2} d \theta=\left\|\check{u}^{(l)}\right\|_{L_{1 / w}^{2}}^{2} .
$$

Hence, the result follows for $l=0, \ldots k$.

\subsection{Fourier-Chebyshev series}

We now consider approximations in terms of Chebyshev polynomials for the solutions of the integral equation (2.12). Specifically, we wish to expand a function $f$ as:

$$
f(x)=\frac{f_{0}}{2} T_{0}(x)+\sum_{n=1}^{\infty} f_{n} T_{n}(x), \quad x \in(-1,1)
$$

with

$$
f_{n}=\frac{2}{\pi}\left(f, T_{n}\right)_{1 / w}, \quad n \in \mathbb{N}_{0} .
$$

The convergence of the Chebyshev series of a function $f$ towards the actual function $f$ is analyzed according to a given norm. In particular, we will study the convergence of approximations in weighted $L^{2}$-norms.

Let $\Pi_{N}((-1,1))$ denote the $(N+1)$-dimensional space spanned by polynomials up to degree $N$ in $(-1,1)$. Define the projection operator

$$
\mathcal{P}_{N}^{T}: L_{1 / w}^{2} \longrightarrow \Pi_{N}((-1,1)),
$$

which approximates $f$ by first kind Chebyshev polynomials $\left\{T_{n}\right\}_{n=0}^{N}$ with

$$
\mathcal{P}_{N}^{T}[f](x)=\frac{f_{0}}{2}+\sum_{n=1}^{N} f_{n} T_{n}(x)
$$

and $f_{n}$ as given in (4.32). We recall the following result found in [21]: 
Theorem 4.1. If $f \in L_{1 / w}^{2}$, then its first kind Chebyshev series expansion converges in the

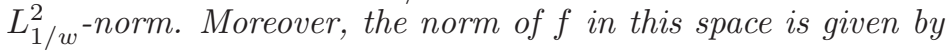

$$
\|f\|_{1 / w}^{2}=\frac{1}{4}\left|f_{0}\right|^{2}+\sum_{n=1}^{\infty}\left|f_{n}\right|^{2} .
$$

Theorem 4.2. Let $u \in W^{k}, k \in \mathbb{N}$ and $N \geq k$. Then the following truncature high-order error estimate holds

$$
\left\|u-\mathcal{P}_{N}^{T} u\right\|_{L_{1 / w}^{2}} \leq N^{-k}\|u\|_{W^{k}}
$$

Proof. Using the mapping (4.22), by Proposition 4.3 one can directly obtain

$$
\left\|u-\mathcal{P}_{N}^{T} u\right\|_{L_{1 / w}^{2}}=\left\|\check{u}-\mathcal{P}_{N}^{\cos } \check{u}\right\|_{L^{2}(I)} \leq N^{-k}\|\check{u}\|_{H_{\mathrm{cos}}^{k}(I)}=N^{-k}\|u\|_{W^{k}}
$$

where the inequality is due to Theorem 3.2 and the last identity to Proposition 4.5.

Remark 4.1. In standard references [7,23], higher-order convergence results are provided for weighted Sobolev spaces for $m \in \mathbb{N}_{0}$ :

$$
H_{1 / w}^{m}:=\left\{u: u^{(\alpha)} \in L_{1 / w}^{2}, \quad 0 \leq|\alpha| \leq m, \quad \alpha \in \mathbb{N}_{0}\right\}
$$

with associated norm

$$
\|u\|_{m, 1 / w}^{2}=\sum_{0 \leq|\alpha| \leq m}\left\|u^{(\alpha)}\right\|_{1 / w}^{2}
$$

for which one can show that the mapping $u \mapsto \check{u}$ is continuous from $H_{1 / w}^{m}$ to $H_{\cos }^{m}(I)$. However, the space $H_{1 / w}^{m}$ is not isomorphic to $H_{\mathrm{cos}}^{m}(I)$ for $m \in \mathbb{N}$ but to a smaller space. Moreover, $W$ contains $H_{1 / w}^{1}$ due to Proposition 4.1 and, for all $m$, the strict continuous inclusion $H_{1 / w}^{m} \subset W^{m}$ holds. This can be understood when comparing the coefficients of the highest order derivatives for both spaces, i.e. $w^{-1 / 2+m}$ for $W^{k}$ and $w^{-1 / 2}$ for $H_{1 / w}^{m}, m \in \mathbb{N}_{0}$.

In view of the above remark, one may be tempted to use only the conditions on the highest order derivative for defining $W^{k}, w^{l} u^{(l)} \in L_{1 / w}^{2}$. Unfortunately, this only works for $k=0,1,2$. For larger $k$, the multiplication of derivatives of $w$ with smaller order derivatives of $u$ blows up.

\subsection{Application to logarithmic integral operators}

As shown in Section 4.2, any function $\varphi \in L_{1 / w}^{2}$ admits a series expansion in terms of $T_{n}$. On the other hand, the logarithmic kernel satisfies the following:

Remark $4.2([10,17])$ For a given $x \in(-1,1)$, the logarithmic kernel admits the expansion on Chebyshev polynomials:

$$
\log \frac{1}{|x-y|}=\log 2+\sum_{n=1}^{\infty} \frac{2}{n} T_{n}(x) T_{n}(y), \quad \forall y \in(-1,1)
$$

as a function in $L_{1 / w}^{2}$. 
The above series converges in the associated weighted norm but not point-wise. $\operatorname{In} \mathcal{C}^{0}((-1,1))$, only Cesàro sums converge. From the orthogonality relation in $L_{1 / w}^{2}$, one obtains that the weighted logarithmic integral operator ${ }^{1)}$ defined as:

$$
\mathcal{L}_{1 / w}[\varphi](x):=\int_{-1}^{1} \log \frac{1}{|x-t|} \frac{\varphi(t)}{\sqrt{1-t^{2}}} d t
$$

has eigenvalues

$$
\lambda_{n}= \begin{cases}\pi \log 2 & n=0 \\ \pi / n & n \geq 1\end{cases}
$$

and eigenfunctions $T_{n}(x), n \geq 0$. With this, we can prove

Proposition 4.6. The operator $\mathcal{L}_{1 / w}: L_{1 / w}^{2} \rightarrow W$ is bounded and continuously invertible, i.e. the Fredholm index zero. If $\vartheta \in W$, the unique solution of the integral equation with purely weighted logarithmic kernel normalized on the interval $(-1,1), \mathcal{L}_{1 / w} \varphi=\vartheta$, is given by

$$
\varphi(x)=\frac{\vartheta_{0}}{2 \pi \log 2} T_{0}(x)+\sum_{n=1}^{\infty} \frac{n}{\pi} \vartheta_{n} T_{n}(x), \quad x \in(-1,1),
$$

where the coefficients $\vartheta_{n}$ are obtained by (4.32).

Proof. The action of $\mathcal{L}_{1 / w}$ over $\varphi$ can be written as the inner product in $L_{1 / w}^{2}$ between the $\operatorname{logarithmic}$ kernel and $\varphi$. Using the Fourier-Chebyshev expansion (4.38), for almost everywhere $x \in(-1,1)$, with $\varphi \in L_{1 / w}^{2}$, we obtain

$$
\mathcal{L}_{1 / w}[\varphi](x)=\log 2\left(T_{0}, \varphi\right)_{1 / w}+\sum_{n=1}^{\infty} \frac{2}{n} T_{n}(x)\left(T_{n}, \varphi\right)_{1 / w},
$$

which clearly shows that $\mathcal{L}_{1 / w} \varphi \in L_{1 / w}^{2}$ as the terms $\left(T_{n}, \varphi\right)_{1 / w}$ are well defined. Since the series (4.38) is convergent in $L_{1 / w}^{2}$ for fixed $y$, for both $x$ and $y$ the series lies in a Hilbert-Schimdt tensor product

$$
L_{1 / w}^{2} \otimes L_{1 / w}^{2} \cong L^{2}\left((-1,1), \frac{d x}{w(x)} ;(-1,1), \frac{d y}{w(y)}\right)
$$

with tensor norm denoted $\|\cdot\|_{1 / w \otimes 1 / w}$. Specifically, we define a bivariate measure

$$
\mu(x, y):=\frac{d x d y}{w(x) w(y)}
$$

under which elements in the tensor product correspond to $L^{2}\left((-1,1)^{2}, \mu\right)$, wherein functions $f(x, y)$ satisfy $\sum_{m, n=0}^{\infty} f_{m n}<\infty$ and $f_{m n}$ are coefficients. These can be approximated by finite sums, and then by taking the limit $N_{x}, N_{y} \rightarrow \infty$ and using Fubini's theorem. Moreover, if we derive in $x$ and, by showing that the residue goes to zero, one can conclude that the derivative $\mathcal{L}_{1 / w}^{\prime}$ is well defined. Furthermore, by relation (4.4) one obtains

$$
\begin{aligned}
\mathcal{L}_{1 / w}^{\prime}[\varphi](x) & =\sum_{n=1}^{\infty} \frac{2}{n} T_{n}^{\prime}(x)\left(T_{n}, \varphi\right)_{1 / w} \\
& =\sum_{n=1}^{\infty} 2 U_{n-1}(x)\left(T_{n}, \varphi\right)_{1 / w}=\sum_{n=0}^{\infty} 2 U_{n}(x)\left(T_{n+1}, \varphi\right)_{1 / w}
\end{aligned}
$$

\footnotetext{
1) Not to be confused with the functional space $L_{1 / w}^{2}$.
} 
implying that $\mathcal{L}_{1 / w}^{\prime} \varphi$ lies in fact in $L_{w}^{2}$. Hence, if $\varphi \in L_{1 / w}^{2}$, then $\mathcal{L}_{1 / w} \varphi \in W$. By the CauchySchwarz inequality, we have

$$
\left|\mathcal{L}_{1 / w} \varphi\right|=\left|\left(\log \frac{1}{|x-\cdot|}, \varphi\right)_{1 / w}\right| \leq\left(\log \frac{1}{|x-\cdot|}, \log \frac{1}{|x-\cdot|}\right)_{1 / w}^{1 / 2}\|\varphi\|_{1 / w} .
$$

Introducing once more the Fourier-Chebyshev series expansion for the logarithm and the orthogonality of $T_{n}$, we obtain

$$
\begin{aligned}
& \quad\left(\log \frac{1}{|x-\cdot|}, \log \frac{1}{|x-\cdot|}\right)_{1 / w}=\pi \log ^{2} 2+\sum_{n=1}^{\infty}\left(\frac{4}{n^{2}} T_{n}^{2}(x)\right) \frac{\pi}{2} \\
& \leq \pi \log ^{2} 2+2 \pi \sum_{n=1}^{\infty} \frac{1}{n^{2}} \leq \pi \log ^{2} 2+\frac{\pi^{2}}{3},
\end{aligned}
$$

proving the boundedness of $\mathcal{L}_{1 / w}$ over $L_{1 / w}^{2}$. Finally, let $\vartheta \in W$. Then, by Theorem 4.1 , we can write both $\varphi$ and $\vartheta$ as Fourier-Chebyshev series (4.31) with coefficients $\varphi_{n}$ and $\vartheta_{n}$ given by (4.32). We take the inner product

$$
\left(\mathcal{L}_{1 / w} \varphi, T_{m}\right)_{1 / w}=\left(\vartheta, T_{m}\right)_{1 / w}=\pi \vartheta_{0} \delta_{0 m}+\frac{\pi}{2} \vartheta_{n} \delta_{n m}
$$

where $\delta_{n m}$ is Kronecker's delta. The left-hand side is equal to

$$
\left(\pi \log 2 \varphi_{0}+\sum_{n=1}^{\infty} \frac{\pi}{n} \varphi_{n} T_{n}(x), T_{m}\right)_{1 / w}=\pi^{2} \log 2 \varphi_{0} \delta_{0 m}+\frac{\pi^{2}}{2 n} \varphi_{n} \delta_{n m} .
$$

Thus,

$$
\varphi_{0}=\frac{\vartheta_{0}}{\pi \log 2} \quad \text { and } \quad \varphi_{n}=\frac{n}{\pi} \vartheta_{n} \quad \text { for } \quad n \geq 1
$$

yielding the stated result.

\subsubsection{Perturbed kernels}

We now perturb the integral equation by adding a sufficiently smooth operator $\mathcal{K}$ :

$$
\left(\mathcal{L}_{1 / w}+\mathcal{K}\right)[\varphi](x)=\int_{-1}^{1}\left[\log \frac{1}{|x-t|}+K(x, t)\right] \frac{\varphi(t)}{\sqrt{1-t^{2}}} d t=f(x)
$$

for $|x| \leq 1$

Lemma 4.2. Let $K(x, t)$ be a Hilbert-Schmidt kernel belonging to the tensor space $L_{1 / w}^{2} \otimes L_{1 / w}^{2}$ with derivative $\partial_{x} K(x, t) \in L_{w}^{2} \otimes L_{1 / w}^{2}$. Both tensor spaces are endowed with the natural HilbertSchmidt norms. Then, the operator $\mathcal{K}: L_{1 / w}^{2} \rightarrow W$ defined as

$$
\mathcal{K}[\varphi](x)=\int_{-1}^{1} K(x, y) \varphi(y) \frac{d y}{\sqrt{1-y^{2}}}
$$

is compact. 
Proof. By the orthogonality relations (4.7) and (4.8), Chebyshev polynomials $T_{n}$ and $U_{n}$ span the spaces $L_{1 / w}^{2}$ and $L_{w}^{2}$, respectively. Thus, $K$ and $\partial_{x} K$ can be represented by FourierChebyshev series of $T_{n}$ and $U_{n}$. By Parseval-Bessel's inequality, we have the Hilbert-Schmidt norms:

$$
\begin{aligned}
\|K(x, y)\|_{1 / w \otimes 1 / w} & =\sum_{n=0}^{\infty} \sum_{m=0}^{\infty}\left|k_{n m}\right|^{2}<\infty, \\
\left\|\partial_{x} K(x, y)\right\|_{w \otimes 1 / w} & =\sum_{n=0}^{\infty} \sum_{m=0}^{\infty} n^{2}\left|k_{n m}\right|^{2}<\infty .
\end{aligned}
$$

For the finite-dimensional operators:

$$
\begin{aligned}
& K_{N}(x, y)=\sum_{n=0}^{N} \sum_{m=0}^{\infty} k_{n m} T_{n}(x) T_{m}(y) \\
& \partial_{x} K_{N}(x, y)=\sum_{n=1}^{N} \sum_{m=0}^{\infty} n k_{n m} U_{n-1}(x) T_{m}(y),
\end{aligned}
$$

it holds

$$
\begin{aligned}
& \lim _{N \rightarrow \infty}\left\|K(x, y)-K_{N}(x, y)\right\|_{1 / w \otimes 1 / w}=\lim _{N \rightarrow \infty} \sum_{n=N+1}^{\infty} \sum_{m=0}^{\infty}\left|k_{n m}\right|^{2}=0, \\
& \lim _{N \rightarrow \infty}\left\|\partial_{x} K(x, y)-\partial_{x} K_{N}(x, y)\right\|_{w \otimes 1 / w}=\lim _{N \rightarrow \infty} \sum_{n=N+1}^{\infty} \sum_{m=0}^{\infty} n^{2}\left|k_{n m}\right|^{2}=0 .
\end{aligned}
$$

Let

$$
\mathcal{K}_{N}[\varphi](x)=\int_{-1}^{1} K_{N}(x, y) \varphi(y) \frac{d y}{\sqrt{1-y^{2}}}=\left(K_{N}(x, \cdot), \varphi\right)_{1 / w},
$$

which is compact since it is of finite range. From the Cauchy-Schwarz inequality, we obtain

$$
\begin{aligned}
\left\|\mathcal{K} \varphi-\mathcal{K}_{N} \varphi\right\|_{W}^{2}= & \left\|\mathcal{K} \varphi-\mathcal{K}_{N} \varphi\right\|_{1 / w}^{2}+\left\|\partial_{x} \mathcal{K} \varphi-\partial_{x} \mathcal{K}_{N} \varphi\right\|_{w}^{2} \\
= & \left(\left|\left(K(x, \cdot)-K_{N}(x, \cdot), \varphi\right)_{1 / w}\right|^{2}, 1(x)\right)_{1 / w} \\
& \quad+\left(\left|\partial_{x}\left(K(x, \cdot)-K_{N}(x, \cdot), \varphi\right)_{1 / w}\right|^{2}, 1(x)\right)_{w} .
\end{aligned}
$$

The first term on the right-hand side of the above is bounded as follows:

$$
\begin{aligned}
& \left(\left|\left(K(x, \cdot)-K_{N}(x, \cdot), \varphi\right)_{1 / w}\right|^{2}, 1(x)\right)_{1 / w} \\
& \leq\left(\|\varphi\|_{1 / w}^{2}\left\|K(x, \cdot)-K_{N}(x, \cdot)\right\|_{1 / w}^{2}, 1(x)\right)_{1 / w} \\
& \leq \pi\left\|K(x, \cdot)-K_{N}(x, \cdot)\right\|_{1 / w \otimes 1 / w}^{2}\|\varphi\|_{1 / w}^{2},
\end{aligned}
$$

whereas for the second term, the bound is

$$
\begin{aligned}
& \left(\left|\partial_{x}\left(K(x, \cdot)-K_{N}(x, \cdot), \varphi\right)_{1 / w}\right|^{2}, 1(x)\right)_{w} \\
& \leq \frac{\pi}{2}\left\|\partial_{x} K(x, \cdot)-\partial_{x} K_{N}(x, \cdot)\right\|_{w \otimes 1 / w}^{2}\|\varphi\|_{1 / w}^{2} .
\end{aligned}
$$


Thus, from (4.54a) and (4.54b), the norm $\left\|\mathcal{K} \varphi-\mathcal{K}_{N} \varphi\right\|_{W}$ tends to zero when $N \rightarrow \infty$ and since the image space $W$ is a Banach space, $\mathcal{K}$ is compact.

Proposition 4.7. Let $\mathcal{K}$ be defined as in Lemma 4.2. Then, the compound operator $\mathcal{L}_{1 / w}+\mathcal{K}:$ $L_{1 / w}^{2} \rightarrow W$ is a Fredholm operator of index zero.

Remark 4.3. Clearly, the same result is found if $\mathcal{K}$ is continuous or weakly continuous over $\mathcal{C}^{0}((-1,1))$.

\subsection{Relation with the original operator $\mathcal{L}$}

The previous results given in weighted $L^{2}$-spaces considered the modified operator $\mathcal{L}_{1 / w}$ and not the original logarithmic operator $\mathcal{L}$. To return to the latter, instead of taking out the factor $w^{-1}$ from $\mathcal{L}_{1 / w}$, we multiply functions in $L_{1 / w}^{2}$ by $w$. By the properties between $L_{1 / w}^{2}$ and $L_{w}^{2}$ described in Proposition 4.1, the statements from Propositions 4.6 and 4.7 can be restated as follows:

Proposition 4.8. The operator $\mathcal{L}=\mathcal{L}_{1 / w} \circ \mathcal{W}: L_{w}^{2} \rightarrow W$ is bounded and continuously invertible. If $g \in W$, the unique solution of the integral equation with logarithmic kernel normalized on the canonical interval (2.12), is given by

$$
\varphi(x)=\frac{g_{0}}{2 \pi \log 2} \frac{T_{0}(x)}{\sqrt{1-x^{2}}}+\sum_{n=1}^{\infty} \frac{n}{\pi} g_{n} \frac{T_{n}(x)}{\sqrt{1-x^{2}}}, \quad x \in(-1,1),
$$

where the coefficients $g_{n}$ are obtained by (4.32).

The following diagram will clarify the point:

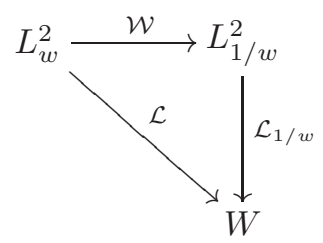

where clearly $\mathcal{L}$ can be interpreted as $\mathcal{L}_{1 / w} \circ \mathcal{W}$. Also, one has following corollary:

Corollary 4.1. Let $\mathcal{K}$ be as in Lemma 4.2 and define $\mathcal{K}_{w}:=\mathcal{K} \circ \mathcal{W}$. The compound operator $\mathcal{L}+\mathcal{K}_{w}: L_{w}^{2} \rightarrow W$ is a Fredholm operator of index zero.

The above observations suggest the use of weighted Chebyshev polynomials to approximate the solutions of logarithmic integral equations to which we turn our discussion.

\section{An Adapted Spectral Galerkin Method}

In Section 2.6 we recalled the continuity and coercivity of the logarithmic integral operator $\mathcal{L}$ defined over $\hat{\Gamma}_{c}=(-1,1) \times\{0\}$ and mapping $\widetilde{H}_{\langle 0\rangle}^{-1 / 2}\left(\hat{\Gamma}_{c}\right)$ onto $H_{*}^{1 / 2}\left(\hat{\Gamma}_{c}\right)$. We now construct approximation spaces on $\hat{\Gamma}_{c}$ :

$$
\mathbb{Q}_{N}(-1,1):=\operatorname{span}\left\{w^{-1} T_{n}\right\}_{n=1}^{N},
$$


and extend their definition over $\Gamma_{c}$, denoted by $\mathbb{Q}_{N}\left(\Gamma_{c}\right)$, through the mapping $x \mapsto \hat{x}$ into $(-1,1)$ :

$$
\hat{x}:=\frac{2\left(x-\bar{x}_{c}\right)}{a_{+}-a_{-}} \quad \text { with } \quad \bar{x}_{c}:=\frac{a_{+}+a_{-}}{2} .
$$

Hence, we can write $\varphi(x)=\hat{\varphi}(\hat{x})$ and approximate $\varphi$ with $\varphi_{N}$ through the weighted truncated Fourier-Chebyshev expansion on the nominal domain:

$$
\varphi_{N}(x)=\hat{\varphi}_{N}(\hat{x}):=w^{-1}(\hat{x}) \sum_{n=1}^{N} \varphi_{n} T_{n}(\hat{x})
$$

where $\varphi_{n}$ are the Fourier-Chebyshev coefficients obtained via $\left(\hat{\varphi}, T_{n}\right)_{1 / w}$. Using standard results [17], one can prove the next proposition:

Proposition 5.1. If $\mathbb{Q}_{N}\left(\Gamma_{c}\right)$ is a closed subspace of $\widetilde{H}_{\langle 0\rangle}^{-1 / 2}\left(\Gamma_{c}\right)$ and if $g$ in $H_{*}^{1 / 2}\left(\Gamma_{c}\right)$, then the Galerkin variational solution $\varphi_{N} \in \mathbb{Q}_{N}\left(\Gamma_{c}\right)$ of

$$
\left\langle\mathcal{L} \varphi_{N}, \varphi_{N}^{t}\right\rangle_{\widetilde{H}^{-1 / 2}\left(\Gamma_{c}\right)}=\left\langle g, \varphi_{N}^{t}\right\rangle_{\widetilde{H}^{-1 / 2}\left(\Gamma_{c}\right)}, \quad \forall \varphi_{N}^{t} \in \mathbb{Q}_{N}\left(\Gamma_{c}\right),
$$

satisfies the following stability condition:

$$
\left\|\varphi_{N}\right\|_{\widetilde{H}^{-1 / 2}\left(\Gamma_{c}\right)} \lesssim\|g\|_{H_{*}^{1 / 2}\left(\Gamma_{c}\right)},
$$

and the error bound:

$$
\left\|\varphi-\varphi_{N}\right\|_{\widetilde{H}^{-1 / 2}\left(\Gamma_{c}\right)} \lesssim \inf _{\psi_{N} \in \mathbb{Q}_{N}\left(\Gamma_{c}\right)}\left\|\varphi-\psi_{N}\right\|_{\widetilde{H}^{-1 / 2}\left(\Gamma_{c}\right)} .
$$

The extension to compactly perturbed operators is immediate. Thus, if besides the principal logarithmic term, continuous functions are introduced in the kernel, the solution scheme remains stable for all $N \geq N_{*}$.

\subsection{Approximation properties of $\mathbb{Q}_{N}\left(\Gamma_{c}\right)$}

We now show that $\mathbb{Q}_{N}\left(\Gamma_{c}\right)$ satisfies the above mentioned approximation property, namely we prove that the spaces are included in $\widetilde{H}_{\langle 0\rangle}^{-1 / 2}\left(\Gamma_{c}\right)$.

Lemma 5.1. The space $\mathbb{Q}_{N}\left(\Gamma_{c}\right)$ is a closed subspace of $\widetilde{H}_{\langle 0\rangle}^{-1 / 2}\left(\Gamma_{c}\right)$.

Proof. Without loss of generality, we consider the canonical segment $\hat{\Gamma}_{c}$. Set $\varphi_{N} \in$ $\mathbb{Q}_{N}(-1,1)$ then $\varphi_{N}=\varphi_{N}^{*} w^{-1}$, with $\varphi_{N}^{*}$ being a polynomial of order $N$. By definition,

$$
\left\|\varphi_{N}\right\|_{\widetilde{H}^{-1 / 2}\left(\hat{\Gamma}_{c}\right)}=\sup _{g \in H^{1 / 2}\left(\hat{\Gamma}_{c}\right)} \frac{\left\langle\varphi_{N}, g\right\rangle_{H^{1 / 2}\left(\hat{\Gamma}_{c}\right)}}{\|g\|_{H^{1 / 2}\left(\hat{\Gamma}_{c}\right)}}=\sup _{g \in H^{1 / 2}\left(\hat{\Gamma}_{c}\right)} \frac{\left\langle w^{-1}, \varphi_{N}^{*} g\right\rangle_{H^{1 / 2}\left(\hat{\Gamma}_{c}\right)}}{\|g\|_{H^{1 / 2}\left(\hat{\Gamma}_{c}\right)}},
$$

and by [12, Theorem 1.4.4.2], it holds

$$
\left\|\varphi_{N}^{*} g\right\|_{H^{1 / 2}\left(\hat{\Gamma}_{c}\right)} \leq\left\|\varphi_{N}^{*}\right\|_{H^{1}\left(\hat{\Gamma}_{c}\right)}\|g\|_{H^{1 / 2}\left(\hat{\Gamma}_{c}\right)},
$$

so that $\varphi_{N}$ belongs to $\widetilde{H}^{-1 / 2}\left(\hat{\Gamma}_{c}\right)$ if $w^{-1}$ does. Denote by $\widetilde{w}$ the extension by zero of $w$ outside $\hat{\Gamma}_{c}$ for which one has the Fourier transform [11]:

$$
\mathcal{F}\left[\tilde{w}^{-1}\right](\xi)=\frac{1}{\sqrt{2 \pi}} \int_{\mathbb{R}} \widetilde{w}^{-1}(x) e^{\imath \xi x} d x=\sqrt{\frac{\pi}{2}} J_{0}(\xi),
$$


where $J_{0}(\xi)$ is the Bessel function of the first kind. Its behavior at infinity is as $\mathcal{O}\left(\xi^{-1 / 2}\right)$. Thus,

$$
\begin{aligned}
\left\|\widetilde{w}^{-1}\right\|_{H^{-1 / 2}(\mathbb{R})}^{2} & =\frac{1}{\sqrt{2 \pi}} \int_{\mathbb{R}}\left(1+\xi^{2}\right)^{-1 / 2}\left[\mathcal{F} \widetilde{w}^{-1}\right]^{2} d \xi \\
& =\frac{\pi}{2 \sqrt{2 \pi}} \int_{\mathbb{R}}\left(1+\xi^{2}\right)^{-1 / 2}\left[J_{0}(\xi)\right]^{2} d \xi<\infty
\end{aligned}
$$

showing the bound in the $H^{-1 / 2}(\mathbb{R})$-norm. Lastly, we need to prove that $\left\langle\varphi_{N}, 1\right\rangle=0$ for all $N$. Since $T_{0}$ is not included in $\mathbb{Q}_{N}(-1,1)$, this comes immediately from the orthogonality relations of Chebyshev polynomials (4.7).

Lemma 5.2. The space $\mathbb{Q}_{\infty}\left(\Gamma_{c}\right)=\lim _{N \rightarrow \infty} \mathbb{Q}_{N}\left(\Gamma_{c}\right)$ is dense in $\widetilde{H}_{\langle 0\rangle}^{-1 / 2}\left(\Gamma_{c}\right)$.

Proof. Since $\mathcal{D}\left(\Gamma_{c}\right)$ is dense in $H^{-1 / 2}\left(\Gamma_{c}\right)$, it is sufficient to show that, for $u \in \mathcal{D}\left(\Gamma_{c}\right)$, we have convergence

$$
\lim _{N \rightarrow \infty} \inf _{u_{N} \in \mathbb{Q}_{N}\left(\Gamma_{c}\right)}\left\|u-u_{N}\right\|_{\widetilde{H}^{-1 / 2}\left(\Gamma_{c}\right)}=0 .
$$

Let us take $u \in \mathcal{D}\left(\Gamma_{c}\right)$, so that

$$
\begin{aligned}
& \left\|u-u_{N}\right\|_{\widetilde{H}^{-1 / 2}\left(\Gamma_{c}\right)} \\
= & \sup _{g \in H^{1 / 2}\left(\Gamma_{c}\right)} \frac{\left\langle u-u_{N}, g\right\rangle_{H^{1 / 2}\left(\Gamma_{c}\right)}}{\|g\|_{H^{1 / 2}\left(\Gamma_{c}\right)}}=\sup _{g \in \mathcal{D}\left(\Gamma_{c}\right)} \frac{\left\langle u-u_{N}, g\right\rangle_{H^{1 / 2}\left(\Gamma_{c}\right)}}{\|g\|_{H^{1 / 2}\left(\Gamma_{c}\right)}},
\end{aligned}
$$

again by density. Since $\left(u-u_{N}\right) g$ belongs to $L^{1}\left(\Gamma_{c}\right)$, the duality can be expressed as the integral

$$
\left\langle u-u_{N}, g\right\rangle_{H^{1 / 2}\left(\Gamma_{c}\right)}=\int_{\Gamma_{c}}\left(u-u_{N}\right) g d x=\int_{\Gamma_{c}} \rho^{1 / 4}\left(u-u_{N}\right) \rho^{-1 / 4} g d x .
$$

where $\rho(x)=\left(x-a_{-}\right)\left(a_{+}-x\right)$ is the "distance" function towards the endpoints of $\Gamma_{c}$. The Cauchy-Schwarz inequality yields

$$
\left|\left\langle u-u_{N}, g\right\rangle_{H^{1 / 2}\left(\Gamma_{c}\right)}\right| \leq\left\|\rho^{1 / 4}\left(u-u_{N}\right)\right\|_{L^{2}\left(\Gamma_{c}\right)}\left\|\rho^{-1 / 4} g\right\|_{L^{2}\left(\Gamma_{c}\right)} .
$$

Now, by Lemma 2.1, it holds

$$
\left\|\rho^{-1 / 4} g\right\|_{L^{2}\left(\Gamma_{c}\right)} \lesssim\|g\|_{H^{1 / 4}\left(\Gamma_{c}\right)} \lesssim\|g\|_{H^{1 / 2}\left(\Gamma_{c}\right)} .
$$

Consequently,

$$
\left\|u-u_{N}\right\|_{\widetilde{H}^{-1 / 2}\left(\Gamma_{c}\right)} \lesssim\left\|\rho^{1 / 4}\left(u-u_{N}\right)\right\|_{L^{2}\left(\Gamma_{c}\right)}=\left\|\rho^{1 / 2} u-u_{N}^{*}\right\|_{L_{1 / w}^{2}},
$$

where $L_{1 / w}^{2}$ is now defined over $\Gamma_{c}$. Since $\rho^{1 / 2} u$ lies in $L_{1 / w}^{2}$, Proposition 4.1 holds and the proof is achieved. 


\subsection{First-order convergence}

As discussed in Section 2.7, the local asymptotic expansion of the Neumann jump density $\varphi$ solution of $(2.13)$ is $(c f .(2.17))$

$$
\varphi=c_{1}^{\prime} \rho^{-1 / 2}+c_{2}^{\prime} \rho^{1 / 2}+\varphi_{R}+\psi \text { on } \Gamma_{c},
$$

where $\varphi_{R} \in \widetilde{H}^{s-3 / 2}\left(\Gamma_{c}\right)$, for $\frac{5}{2}<s<\frac{7}{2}$, and

$$
\psi=\rho^{-1 / 2} \sum_{ \pm}\left(c_{1 \pm}^{\prime}\left(\eta_{ \pm}-1\right)+c_{2 \pm}^{\prime}\left(\eta_{ \pm}-1\right) \rho\right) \quad \text { on } \Gamma_{c}
$$

with real coefficients $c_{i \pm}, i=1,2$. The main result of this section is the next proposition, whose proof is based on a series of Lemmas provided in the Appendix.

Proposition 5.2. Let $\varphi \in \widetilde{H}_{\langle 0\rangle}^{-1 / 2}\left(\Gamma_{c}\right)$ admit the splitting (5.15) for $\frac{5}{2}<s<\frac{7}{2}$. Then, there exist $N_{*} \in \mathbb{N}$ and $\varphi_{N} \in \mathbb{Q}_{N}\left(\Gamma_{c}\right)$ such that

$$
\left\|\varphi-\varphi_{N}\right\|_{\widetilde{H}^{-1 / 2}\left(\Gamma_{c}\right)} \lesssim N^{-1}\left(\left\|\varphi_{R}\right\|_{\widetilde{H}^{s-3 / 2}\left(\Gamma_{c}\right)}+\sum_{ \pm}\left(\left|c_{1 \pm}^{\prime}\right|+\left|c_{2 \pm}^{\prime}\right|\right)\right) \quad \forall N \geq N_{*} .
$$

Proof. The key idea is to reduce the error bound from $\varphi-\varphi_{N}$ to the one on regular parts. Without loss of generality, we consider the canonical segment $\hat{\Gamma}_{c}$. Recalling the splitting (5.15) of $\varphi$,

$$
\varphi=\rho^{-1 / 2}\left(c_{1}^{\prime}+c_{2}^{\prime} \rho+\varphi_{R}^{*}+\psi^{*}\right),
$$

we look for its approximation $\varphi_{N}$ with $N \geq 2$ in the form

$$
\varphi_{N}=\rho^{-1 / 2}\left(c_{1}^{\prime}+c_{2}^{\prime} \rho+\varphi_{R, N}^{*}+\psi_{N}^{*}\right)
$$

where the coefficients $c_{i}^{\prime}, i=1,2$, are the same as for $\varphi$ given in (5.15) and $\varphi_{R, N}^{*}:=\mathcal{P}_{N}^{T} \varphi_{R}^{*}$ (resp. $\psi_{N}^{*}:=\mathcal{P}_{N}^{T} \psi^{*}$ ) is the $L_{1 / w^{2}}^{2}$-projection on the set of polynomials of degree $\leq N$ of $\varphi_{R}^{*}$ (resp. $\psi^{*}$ ) given by Theorem 4.2. By construction, $\varphi_{N}^{*}=\rho^{1 / 2} \varphi_{N}$ is a polynomial of degree $N$, hence it is a linear combination of Chebyshev polynomials $T_{n}$ for $n \in\{0,1, \cdots, N\}$. Therefore, in view of the definition of $\mathbb{Q}_{N}(-1,1)$, we need to show that

$$
\int_{-1}^{1} \varphi_{N}^{*}(x) w^{-1}(x) d x=0 .
$$

By definition, $\varphi$ satisfies $(\varphi, 1)_{\hat{\Gamma}_{c}}=0$. Since $\rho^{1 / 2}=w$, we deduce that $\varphi^{*}=\rho^{1 / 2} \varphi$ satisfies $\left(\varphi^{*}, 1\right)_{1 / w}=0$. As

$$
\varphi^{*}=c_{1}^{\prime}+c_{2}^{\prime} \rho+\varphi_{R}^{*}+\psi^{*}
$$

we deduce that

$$
\int_{-1}^{1}\left(\varphi_{R}^{*}(x)+\psi^{*}(x)\right) w^{-1}(x) d x=-\int_{-1}^{1}\left(c_{1}^{\prime}+c_{2}^{\prime} \rho(x)\right) w^{-1}(x) d x .
$$

Now, we make use of the fact that $\varphi_{R, N}^{*}\left(\right.$ resp. $\left.\psi_{N}^{*}\right)$ is the $L_{1 / w}^{2}$ projection on the set of polynomials of degree $\leq N$ of $\varphi_{R}^{*}\left(\right.$ resp. $\left.\psi^{*}\right)$ and, therefore

$$
\int_{-1}^{1}\left(\varphi_{R, N}^{*}(x)+\psi_{N}^{*}(x)\right) w^{-1}(x) d x=\int_{-1}^{1}\left(\varphi_{R}^{*}(x)+\psi^{*}(x)\right) w^{-1}(x) d x .
$$


as one is a polynomial of degree $\leq N$. Hence, by using (5.20), we obtain

$$
\int_{-1}^{1}\left(\varphi_{R, N}^{*}(x)+\psi_{N}^{*}(x)\right) w^{-1}(x) d x=-\int_{-1}^{1}\left(c_{1}^{\prime}+c_{2}^{\prime} \rho(x)\right) w^{-1}(x) d x
$$

which is nothing else than (5.19). This shows that $\varphi_{N}$ belongs to $\mathbb{Q}_{N}(-1,1)$. Also, by construction, it holds

$$
\varphi-\varphi_{N}=\rho^{-1 / 2}\left(\varphi_{R}^{*}-\varphi_{R, N}^{*}+\psi^{*}-\psi_{N}^{*}\right)
$$

and we can apply Lemma A.4 to obtain

$$
\left\|\varphi-\varphi_{N}\right\|_{\widetilde{H}^{-1 / 2}\left(\Gamma_{c}\right)} \lesssim\left\|\varphi_{R}^{*}-\varphi_{R, N}^{*}\right\|_{L_{1 / w}^{2}}+\left\|\psi^{*}-\psi_{N}^{*}\right\|_{L_{1 / w}^{2}} .
$$

By Theorem 4.2, we deduce that

$$
\left\|\varphi-\varphi_{N}\right\|_{\widetilde{H}^{-1 / 2}\left(\Gamma_{c}\right)} \lesssim N^{-1}\left(\left\|\varphi_{R}^{*}\right\|_{W}+\left\|\psi^{*}\right\|_{W}\right),
$$

and we conclude thanks to Lemmas A.2 and A.3.

\subsection{Higher-order convergence}

Our goal now is to obtain higher-order convergence for the solution of the integral equation (2.12) results for smoother data. As before, if $g$ is smooth enough, then for $m \geq 1$, the solution $u$ admits the local decomposition at the endpoints [12]:

$$
\eta_{ \pm} u\left(r_{ \pm}, \theta_{ \pm}\right)=\eta_{ \pm} \sum_{i=0}^{m} c_{i \pm} r_{ \pm}^{\frac{1}{2}+i} \sin \left\{\left(\frac{1}{2}+i\right) \theta_{ \pm}\right\}+u_{R \pm}
$$

where $u_{R \pm} \in H_{\text {loc }}^{s}(\Omega)$ with $m+\frac{3}{2}<s<m+\frac{5}{2}$. Hence the normal derivative jump along $\Gamma_{c}$ behaves locally as

$$
\eta_{ \pm} \varphi=\eta_{ \pm}\left[\frac{\partial u}{\partial n}\right]=\sum_{i=0}^{m} c_{i \pm}^{\prime} \eta_{ \pm} r^{i-\frac{1}{2}}+\varphi_{R \pm}
$$

with $\varphi_{R \pm} \in \widetilde{H}^{s-\frac{3}{2}}\left(\Gamma_{c}\right)$. In order to replace $r_{ \pm}$by $\rho$, we first consider $r_{-}$over $\Gamma_{c}$ and use that

$$
r_{-}^{i-\frac{1}{2}}(x)=\rho^{i-\frac{1}{2}}(x)\left(a_{+}-x\right)^{\frac{1}{2}-i}, \quad x \in\left(a_{-}, a_{+}\right) .
$$

Since $\left(a_{+}-x\right)^{\frac{1}{2}-i}$ is smooth near $x=a_{-}$, it can be written as

$$
\left(a_{+}-x\right)^{\frac{1}{2}-i}=p_{i K}(x)+r_{i K}(x),
$$

where $p_{i K}(x)=\sum_{k=0}^{K} a_{i k}\left(x+a_{-}\right)^{k}$ is the Taylor expansion of $\left(a_{+}-x\right)^{\frac{1}{2}-i}$ at $x=a_{-}$truncated at the order $K$ and $r_{i K}$ is the residue. If $K+i>s-\frac{7}{2}$, we deduce that

$$
\rho^{i-\frac{1}{2}} r_{i K} \in \widetilde{H}^{s-\frac{3}{2}}\left(\Gamma_{c}\right)
$$

Due to the constraint $m+\frac{3}{2}<s<m+\frac{5}{2}$, we can chose $K=m-1$. By employing a similar argument at $x=a_{+}$, we deduce that

$$
\eta_{ \pm} \varphi=\eta_{ \pm} \rho^{-\frac{1}{2}} p_{ \pm}+\varphi_{R \pm},
$$


where $p_{ \pm}$is a polynomial of degree smaller or equal to $3 m-1$ and $\varphi_{R \pm} \in \widetilde{H}^{s-\frac{3}{2}}\left(\Gamma_{c}\right)$.

As in Section 2.7, we can now state the decomposition:

$$
\varphi=\rho^{-\frac{1}{2}} p+\varphi_{R}+\psi,
$$

where $p$ is polynomial of degree less or equal to $3 m-1, \varphi_{R} \in \widetilde{H}^{s-\frac{3}{2}}\left(\Gamma_{c}\right)$ and

$$
\psi:=\rho^{-\frac{1}{2}} \sum_{ \pm}\left(1-\eta_{ \pm}\right) p_{ \pm} .
$$

Proposition 5.3. Let $\varphi \in \widetilde{H}_{\langle 0\rangle}^{-1 / 2}\left(\Gamma_{c}\right)$ admit the decomposition (5.24) with $\varphi_{R} \in \widetilde{H}^{s-\frac{3}{2}}\left(\Gamma_{c}\right)$ for $m+\frac{3}{2}<s<m+\frac{5}{2}$. Then, there exist $N_{*} \in \mathbb{N}, \varphi_{N} \in \mathbb{Q}_{N}\left(\Gamma_{c}\right)$ and a positive constant $c(m)$ depending only on $m$ such that

$$
\left\|\varphi-\varphi_{N}\right\|_{\widetilde{H}^{-1 / 2}\left(\Gamma_{c}\right)} \leq c(m) N^{-m}\left(\left\|\varphi_{R}\right\|_{\widetilde{H}^{s-1 / 2}\left(\Gamma_{c}\right)}+\sum_{ \pm} \sum_{i=0}^{m}\left|c_{i \pm}^{\prime}\right|\right)
$$

for all $N \geq N_{*}$.

Proof. The proof is the same as the one of Proposition 5.2. Here, we make use of Lemmas A.5 and A.6.

For numerical experiments, instead of verifying the inequality (5.26), we use the following equivalent result.

Corollary 5.1. We can verify Propositions 5.2 and 5.3 by finding, for each $m \geq 1$, an $N_{*} \in \mathbb{N}$ such that

$$
\left\|\varphi-\varphi_{N}\right\|_{\widetilde{H}^{-1 / 2}(\Gamma)} \lesssim N^{-m}\left(\left\|\varphi_{R}^{*}+\psi^{*}\right\|_{W^{m}}\right), \quad \forall N \geq N_{*},
$$

where $\|u\|_{W^{m}}^{2}=\sum_{l=0}^{m}\left\|\mathrm{D}^{l} u\right\|_{1 / w}^{2}$.

Proof. Recall the asymptotic decomposition

$$
\varphi=c_{1} \rho^{-1 / 2}+c_{2} \rho^{1 / 2}+\varphi_{R}+\psi .
$$

We also have

$$
\varphi_{N}=c_{1} \rho^{-1 / 2}+c_{2} \rho^{1 / 2}+\varphi_{R N}+\psi_{N} .
$$

Now, by construction we have

$$
\varphi-\varphi_{N}=\rho^{-1 / 2}\left(\varphi_{R}^{*}-\varphi_{R N}^{*}+\psi^{*}-\psi_{N}^{*}\right),
$$

and we can apply Lemma A.4 to obtain

$$
\left\|\varphi-\varphi_{N}\right\|_{\widetilde{H}^{-1 / 2}(\Gamma)} \lesssim\left\|\varphi_{R}^{*}+\psi^{*}-\varphi_{R N}^{*}-\psi_{N}^{*}\right\|_{L_{1 / w}^{2}} .
$$

Finally, by Theorem 4.2, it holds

$$
\left\|\varphi-\varphi_{N}\right\|_{\widetilde{H}^{-1 / 2}(\Gamma)} \lesssim N^{-m}\left\|\varphi_{R}^{*}+\psi^{*}\right\|_{W^{m}} .
$$

Verification of Propositions 5.2 and 5.3 comes from Lemmas A.3 and A.6, respectively. Indeed, by using the triangle inequality, we obtain

$$
\begin{aligned}
\left\|\varphi-\varphi_{N}\right\|_{\widetilde{H}^{-1 / 2}(\Gamma)} & \lesssim N^{-m}\left\|\varphi_{R}^{*}+\psi^{*}\right\|_{W^{m}} \lesssim N^{-m}\left(\left\|\varphi_{R}^{*}\right\|_{W^{m}}+\left\|\psi^{*}\right\|_{W^{m}}\right) \\
& \lesssim N^{-m}\left(\left\|\varphi_{R}^{*}\right\|_{W^{m}}+\sum_{ \pm}\left(\left|c_{1}^{\prime} \pm\right|+\left|c_{2}^{\prime} \pm\right|\right)\right) .
\end{aligned}
$$

Lastly, as in Remark 3.2, exponential convergence can be achieved if the solution $\varphi$ is analytic. 


\section{Numerical Results}

In what follows, we present several numerical experiments to validate our findings. Let $Q$ denote the number of quadrature points, and $N$ the number of spectral degrees of freedom (dofs), with specific values for both quantities given for each test. We consider two error norms:

(i) $W^{m}$-norms, for $m \leq 3$, for which the corresponding $\mathrm{D}^{l}$ derivatives are calculated analytically; and,

(ii) $\widetilde{H}^{-1 / 2}$-norms, defined over $\hat{\Gamma}_{c}$, obtained through the energy norm related to the weakly singular operator $\mathcal{L}$.

For the latter, Galerkin orthogonality yields

$$
\begin{aligned}
\left\|\varphi-\varphi_{N}\right\|_{\widetilde{H}^{-1 / 2}\left(\hat{\Gamma}_{c}\right)}^{2} \approx\left\langle\mathcal{L}\left(\varphi-\varphi_{N}\right),\left(\varphi-\varphi_{N}\right)\right\rangle_{\widetilde{H}^{-1 / 2}\left(\hat{\Gamma}_{c}\right)} \\
=\langle\mathcal{L} \varphi, \varphi\rangle_{\widetilde{H}^{-1 / 2}\left(\hat{\Gamma}_{c}\right)}-\left\langle\mathcal{L} \varphi_{N}, \varphi_{N}\right\rangle_{\widetilde{H}^{-1 / 2}\left(\hat{\Gamma}_{c}\right)}, \quad \forall \varphi \in \widetilde{H}_{\langle 0\rangle}^{-1 / 2}\left(\hat{\Gamma}_{c}\right),
\end{aligned}
$$

and where the first term is calculated by spectral quadrature using an overkill numerical solution $\varphi^{o k}$ of $N_{o k}$ spectral dofs such that $N_{o k} \gg N$. Then, by defining $\underline{\varphi}_{N}$ as the vector of associated Chebyshev coefficients, and $\underline{\underline{\mathcal{L}}}$ as the discrete weakly singular spectral diagonal matrix, (6.1) boils down to

$$
\left\|\varphi-\varphi_{N}\right\|_{\widetilde{H}^{-1 / 2}\left(\hat{\Gamma}_{c}\right)}^{2} \approx\left(\underline{\varphi}^{o k}-\underline{\varphi}_{N}\right)^{T} \underline{\underline{\mathcal{L}}}\left(\underline{\varphi}^{o k}-\underline{\varphi}_{N}\right) .
$$

The rationale behind this comparison between $W^{m}$ and $\widetilde{H}^{-1 / 2}$-error norms comes from Corollary 5.1 as it is difficult to compute $\widetilde{H}^{s}$-norms for $s \geq 1 / 2$.

\subsection{Converge results for approximation of elements in $\widetilde{H}^{-1 / 2}\left(\hat{\Gamma}_{c}\right)$}

Tables 6.1-6.3 show convergence results obtained for the approximation of different choices of $\varphi(x)$ :

$$
x w^{-1}, \quad w^{-1}\left(4 x^{3}-3 x\right), \quad w^{-1} \sin x, \quad w \cos x-w^{-1} \sin x, \quad \sin (2 \pi x) .
$$

We clearly observe the described convergence rates of Corollary 5.1. For all examples, the values for $Q$ and $N_{o k}$ remained the same in order to compare their behavior at a given precision. Although one would naively expect that the accuracy of $\varphi^{o k}$ would improve when increasing $N_{o k}$, we actually observe that after a certain threshold it introduces additional numerical error. This phenomenon is customary in high-order methods. Finally, it is worth mentioning that the even Chebyshev coefficients of the function $\varphi=\sin (2 \pi x)$ are zero, therefore, Table 6.3 only shows the results for the odd numbers of degrees of freedom.

\subsection{Convergence results for the Laplace problem with Dirichlet boundary condi- tions}

From Proposition 4.8, if we consider $g \in W$ as our right-hand side, then the numerical approximation of $\varphi \in \widetilde{H}_{\langle 0\rangle}^{-1 / 2}\left(\hat{\Gamma}_{c}\right)$ in $\mathbb{Q}_{N}(-1,1)$ is given by

$$
\varphi_{N}(x)=w^{-1}(x) \sum_{n=1}^{N} \vartheta_{n} T_{n}(x) .
$$

We run numerical experiments by using different functions $g \in W$ as our right-hand side. 
Table 6.1: Fist-order and higher-order convergence results with $N_{o k}=Q=100$.
(a) $\varphi(x)=x w^{-1}(x)$.
(b) $\varphi(x)=\left(4 x^{3}-3 x\right) w^{-1}(x)$.

\begin{tabular}{|c|c|c|c|c|}
\hline $\mathrm{N}$ & $\left\|\varphi-\varphi_{N}\right\|_{\widetilde{H}^{-1 / 2}}$ & $\begin{array}{c}N^{-m} \\
m=1\end{array}$ & $\begin{array}{c}\| \varphi_{R}^{*}+\psi^{*} \\
m=2\end{array}$ & $\|_{W^{m}}$ \\
\hline 2 & $4.82 \mathrm{e}-15$ & $8.86 \mathrm{e}-01$ & $5.43 \mathrm{e}-01$ & $1.75 \mathrm{e}-01$ \\
\hline 3 & $4.82 \mathrm{e}-15$ & $5.91 \mathrm{e}-01$ & $2.41 \mathrm{e}-01$ & $3.46 \mathrm{e}-02$ \\
\hline 4 & $4.81 \mathrm{e}-15$ & $4.43 \mathrm{e}-01$ & $1.36 \mathrm{e}-01$ & $1.09 \mathrm{e}-02$ \\
\hline 5 & $4.80 \mathrm{e}-15$ & $3.54 \mathrm{e}-01$ & $8.68 \mathrm{e}-02$ & $4.48 \mathrm{e}-03$ \\
\hline
\end{tabular}

\begin{tabular}{|c|c|c|c|c|}
\hline $\mathrm{N}$ & $\left\|\varphi-\varphi_{N}\right\|_{\widetilde{H}^{-1 / 2}}$ & 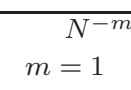 & $\begin{array}{c}\| \varphi_{R}^{*}+\psi^{*} \\
m=2\end{array}$ & $\begin{array}{l}\|_{W^{m}} \\
\quad m=3\end{array}$ \\
\hline 2 & $1.29 \mathrm{e}+00$ & $2.17 \mathrm{e}+00$ & $3.04 \mathrm{e}+00$ & $4.50 \mathrm{e}+00$ \\
\hline 3 & $0.00 \mathrm{e}+00$ & $1.45 \mathrm{e}+00$ & $1.35 \mathrm{e}+00$ & $1.33 \mathrm{e}+00$ \\
\hline 4 & $0.00 \mathrm{e}+00$ & $1.09 \mathrm{e}+00$ & $7.59 \mathrm{e}-01$ & $5.62 \mathrm{e}-01$ \\
\hline 5 & $0.00 \mathrm{e}+00$ & $8.68 \mathrm{e}-01$ & $4.86 \mathrm{e}-01$ & $2.88 \mathrm{e}-01$ \\
\hline
\end{tabular}

Table 6.2: Fist-order and higher-order convergence results with $N_{o k}=Q=100$.
(a) $\varphi(x)=\sin (x) w^{-1}(x)$.
(b) $\varphi(x)=\cos (x) w(x)-\sin (x) w^{-1}(x)$.

\begin{tabular}{|c|c|c|c|c|}
\hline $\mathrm{N}$ & $\left\|\varphi-\varphi_{N}\right\|_{\widetilde{H}^{-1 / 2}}$ & $\begin{array}{c}N^{-m} \\
m=1\end{array}$ & $\begin{array}{c}\| \varphi_{R}^{*}+\psi^{*} \\
m=2\end{array}$ & $\begin{array}{l}\|_{W^{m}} \\
m=3\end{array}$ \\
\hline 2 & $5.02 \mathrm{e}-02$ & $7.84 \mathrm{e}-01$ & $4.92 \mathrm{e}-01$ & $3.27 \mathrm{e}-01$ \\
\hline 4 & $4.96 \mathrm{e}-04$ & $3.92 \mathrm{e}-01$ & $1.23 \mathrm{e}-01$ & $4.09 \mathrm{e}-02$ \\
\hline 6 & $2.52 \mathrm{e}-06$ & $2.61 \mathrm{e}-01$ & $5.46 \mathrm{e}-02$ & $1.21 \mathrm{e}-02$ \\
\hline 8 & 7.77e-09 & $1.96 \mathrm{e}-01$ & $3.07 \mathrm{e}-02$ & $5.11 \mathrm{e}-03$ \\
\hline 10 & $1.60 \mathrm{e}-11$ & $1.57 \mathrm{e}-01$ & $1.97 \mathrm{e}-02$ & $2.62 \mathrm{e}-03$ \\
\hline 12 & $2.42 \mathrm{e}-14$ & $1.31 \mathrm{e}-01$ & $1.37 \mathrm{e}-02$ & $1.51 \mathrm{e}-03$ \\
\hline 14 & $4.05 \mathrm{e}-15$ & $1.12 \mathrm{e}-01$ & $1.00 \mathrm{e}-02$ & $9.53 \mathrm{e}-04$ \\
\hline
\end{tabular}

\begin{tabular}{|c|c|c|c|c|}
\hline $\mathrm{N}$ & $\left\|\varphi-\varphi_{N}\right\|_{\widetilde{H}^{-1 / 2}}$ & $\begin{array}{c}N^{-m} \\
m=1\end{array}$ & $\begin{array}{c}\varphi_{R}^{*}+\psi^{*} \\
m=2\end{array}$ & $\|_{m}{ }^{m}=3$ \\
\hline 2 & $8.80 \mathrm{e}-02$ & $1.08 \mathrm{e}+00$ & $1.55 \mathrm{e}+00$ & $2.39 \mathrm{e}+00$ \\
\hline 4 & $1.37 \mathrm{e}-03$ & $5.39 \mathrm{e}-01$ & $3.87 \mathrm{e}-01$ & $2.98 \mathrm{e}-01$ \\
\hline 6 & $9.47 \mathrm{e}-06$ & $3.59 \mathrm{e}-01$ & $1.72 \mathrm{e}-01$ & $8.83 \mathrm{e}-02$ \\
\hline 8 & $3.70 \mathrm{e}-08$ & $2.69 \mathrm{e}-01$ & $9.67 \mathrm{e}-02$ & $3.73 \mathrm{e}-02$ \\
\hline 10 & $9.23 \mathrm{e}-11$ & $2.16 \mathrm{e}-01$ & $6.19 \mathrm{e}-02$ & $1.91 \mathrm{e}-02$ \\
\hline 12 & $1.60 \mathrm{e}-13$ & $1.80 \mathrm{e}-01$ & $4.30 \mathrm{e}-02$ & $1.10 \mathrm{e}-02$ \\
\hline 14 & $4.01 \mathrm{e}-15$ & $1.54 \mathrm{e}-01$ & $3.16 \mathrm{e}-02$ & $6.95 \mathrm{e}-03$ \\
\hline
\end{tabular}

Table 6.3: Fist-order and higher-order convergence results for $\varphi(x)=\sin (2 \pi x)$ with $N_{o k}=Q=100$.

\begin{tabular}{|c|cccc|}
\hline $\mathrm{N}$ & $\left\|\varphi-\varphi_{N}\right\|_{\widetilde{H}^{-1 / 2}}$ & $N^{-1}\left\|\varphi_{R}^{*}+\psi^{*}\right\|_{W}$ & $N^{-2}\left\|\varphi_{R}^{*}+\psi^{*}\right\|_{W^{2}}$ & $N^{-3}\left\|\varphi_{R}^{*}+\psi^{*}\right\|_{W^{3}}$ \\
\hline 2 & $7.9131 \mathrm{e}-01$ & $2.4824 \mathrm{e}+00$ & $7.5163 \mathrm{e}+00$ & $2.4557 \mathrm{e}+01$ \\
3 & $7.8778 \mathrm{e}-01$ & $1.6549 \mathrm{e}+00$ & $3.3406 \mathrm{e}+00$ & $7.2761 \mathrm{e}+00$ \\
5 & $2.6804 \mathrm{e}-01$ & $9.9297 \mathrm{e}-01$ & $1.2026 \mathrm{e}+00$ & $1.5716 \mathrm{e}+00$ \\
7 & $4.3338 \mathrm{e}-02$ & $7.0926 \mathrm{e}-01$ & $6.1358 \mathrm{e}-01$ & $5.7276 \mathrm{e}-01$ \\
9 & $4.2260 \mathrm{e}-03$ & $5.5165 \mathrm{e}-01$ & $3.7118 \mathrm{e}-01$ & $2.6949 \mathrm{e}-01$ \\
11 & $2.7928 \mathrm{e}-04$ & $4.5135 \mathrm{e}-01$ & $2.4847 \mathrm{e}-01$ & $1.4760 \mathrm{e}-01$ \\
13 & $1.3417 \mathrm{e}-05$ & $3.8191 \mathrm{e}-01$ & $1.7790 \mathrm{e}-01$ & $8.9420 \mathrm{e}-02$ \\
15 & $4.9137 \mathrm{e}-07$ & $3.3099 \mathrm{e}-01$ & $1.3362 \mathrm{e}-01$ & $5.8209 \mathrm{e}-02$ \\
17 & $1.4199 \mathrm{e}-08$ & $2.9205 \mathrm{e}-01$ & $1.0403 \mathrm{e}-01$ & $3.9987 \mathrm{e}-02$ \\
19 & $3.3240 \mathrm{e}-10$ & $2.6131 \mathrm{e}-01$ & $8.3283 \mathrm{e}-02$ & $2.8642 \mathrm{e}-02$ \\
21 & $6.4375 \mathrm{e}-12$ & $2.3642 \mathrm{e}-01$ & $6.8175 \mathrm{e}-02$ & $2.1213 \mathrm{e}-02$ \\
23 & $1.0491 \mathrm{e}-13$ & $2.1586 \mathrm{e}-01$ & $5.6834 \mathrm{e}-02$ & $1.6147 \mathrm{e}-02$ \\
25 & $1.4590 \mathrm{e}-15$ & $1.9859 \mathrm{e}-01$ & $4.8104 \mathrm{e}-02$ & $1.2573 \mathrm{e}-02$ \\
\hline \multicolumn{5}{|c}{} \\
\hline
\end{tabular}

- Example 1. Let $g(x)=\sin (2 \pi x)$. The corresponding analytical solution is

$$
\varphi(x)=\sum_{n=0}^{\infty} \frac{2 n}{\pi} a_{2 n} T_{2 n}(x), \quad x \in(-1,1),
$$

where $a_{2 n}=2 J_{n}(2 \pi) \sin \left(\frac{n \pi}{2}\right)$. Figure $6.1($ a $)$ shows the obtained errors. They are also detailed in Table 6.4, from where we observe that numerical precision is achieved with $N=27$. 
Table 6.4: Convergence results for solving $\mathcal{L} \varphi=g$ using an overkill $\varphi^{o k}$ of $N_{o k}=100$ dofs.

(a) $g(x)=\sin (2 \pi x)$

\begin{tabular}{|c|c|c|}
\hline $\mathrm{N}$ & $\left\|\varphi^{o k}-\varphi_{N}\right\|_{1 / w}$ & $\left\|\varphi^{o k}-\varphi_{N}\right\|_{\widetilde{H}-1 / 2}$ \\
\hline 2 & $1.0204 \mathrm{e}+01$ & $1.3259 \mathrm{e}+00$ \\
3 & $1.1155 \mathrm{e}+01$ & $1.3240 \mathrm{e}+00$ \\
5 & $9.5472 \mathrm{e}+00$ & $6.0238 \mathrm{e}-01$ \\
7 & $2.4860 \mathrm{e}+00$ & $1.2445 \mathrm{e}-01$ \\
9 & $3.3971 \mathrm{e}-01$ & $1.4810 \mathrm{e}-02$ \\
11 & $2.9248 \mathrm{e}-02$ & $1.1561 \mathrm{e}-03$ \\
13 & $1.7478 \mathrm{e}-03$ & $6.4075 \mathrm{e}-05$ \\
15 & $7.7073 \mathrm{e}-05$ & $2.6592 \mathrm{e}-06$ \\
17 & $2.6174 \mathrm{e}-06$ & $8.5877 \mathrm{e}-08$ \\
19 & $7.0661 \mathrm{e}-08$ & $2.2220 \mathrm{e}-09$ \\
21 & $1.5543 \mathrm{e}-09$ & $4.7131 \mathrm{e}-11$ \\
23 & $2.8407 \mathrm{e}-11$ & $8.3483 \mathrm{e}-13$ \\
25 & $4.3780 \mathrm{e}-13$ & $1.2539 \mathrm{e}-14$ \\
\hline
\end{tabular}

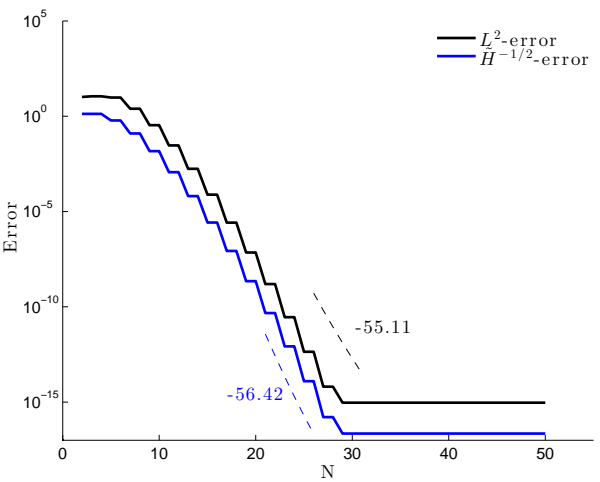

(a) $g(x)=\sin (2 \pi x)$ (b) $g(x)=\exp (x)$

\begin{tabular}{|c|c|c|}
\hline $\mathrm{N}$ & $\left\|\varphi^{o k}-\varphi_{N}\right\|_{1 / w}$ & $\left\|\varphi^{o k}-\varphi_{N}\right\|_{\widetilde{H}-1 / 2}$ \\
\hline 2 & $7.6387 \mathrm{e}-01$ & $5.4857 \mathrm{e}-02$ \\
3 & $1.2343 \mathrm{e}-01$ & $7.7896 \mathrm{e}-03$ \\
4 & $1.5122 \mathrm{e}-02$ & $8.6199 \mathrm{e}-04$ \\
5 & $1.4898 \mathrm{e}-03$ & $7.8133 \mathrm{e}-05$ \\
6 & $1.2265 \mathrm{e}-04$ & $5.9970 \mathrm{e}-06$ \\
7 & $8.6701 \mathrm{e}-06$ & $3.9911 \mathrm{e}-07$ \\
8 & $5.3686 \mathrm{e}-07$ & $2.3445 \mathrm{e}-08$ \\
9 & $2.9571 \mathrm{e}-08$ & $1.2326 \mathrm{e}-09$ \\
10 & $1.4667 \mathrm{e}-09$ & $5.8638 \mathrm{e}-11$ \\
11 & $6.6157 \mathrm{e}-11$ & $2.5474 \mathrm{e}-12$ \\
12 & $2.7381 \mathrm{e}-12$ & $1.0183 \mathrm{e}-13$ \\
13 & $1.0399 \mathrm{e}-13$ & $3.7806 \mathrm{e}-15$ \\
14 & $4.7499 \mathrm{e}-15$ & $3.2121 \mathrm{e}-16$ \\
\hline
\end{tabular}

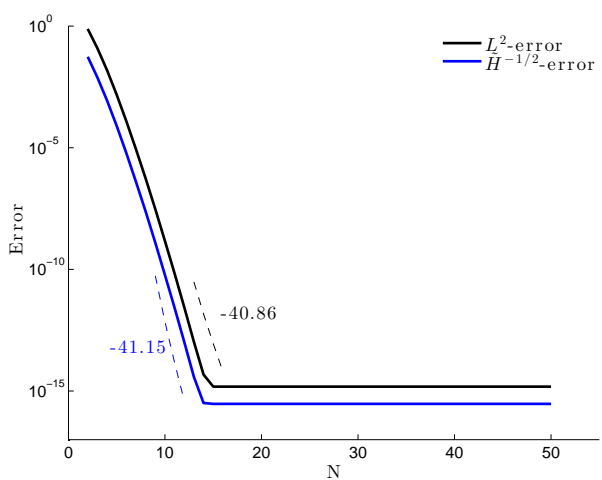

(b) $g(x)=e^{x}$

Fig. 6.2. Convergence results for $\varphi$ solution of $\mathcal{L} \varphi=g$.

- Example 2. Now set $g(x)=\exp (x)$. The exact solution is

$$
\varphi(x)=\frac{J_{0}(i)}{2 \pi \log (2)} T_{0}(x)+2 \sum_{n=1}^{\infty} \frac{n}{\pi} i^{n} J_{n}(-i) T_{n}(x), \quad x \in(-1,1) .
$$

The resulting convergence is summarized in Table 6.4 and it is also contained in Figure 6.1(b). This time we obtained numerical precision with $N=14$.

For both examples, it can be seen that the solutions converge exponentially in $N$ as can be expected from the analyticity of the source terms $g$.

\subsection{Helmholtz problem}

We now consider the weakly singular operator for the Helmholtz problem. In this case, the operator can be decomposed into singular and compact terms, with the singular operator being equal to the Laplace case. We provide a comparison between solutions obtained via 
the presented spectral discretization basis and traditional low-order boundary elments or $h$ refinement at two different wavenumbers.

Table 6.5 shows absolute and relative $\widetilde{H}^{-1 / 2}\left(\hat{\Gamma}_{c}\right)$-errors obtained when using again an overkill numerical solution $\varphi^{o k}$ as reference. The relative error is measured as

$$
\text { rel. error }=\frac{\left\|\varphi^{o k}-\varphi_{N}\right\|_{\widetilde{H}^{-1 / 2}\left(\hat{\Gamma}_{c}\right)}}{\left\|\varphi^{o k}\right\|_{\widetilde{H}^{-1 / 2}\left(\hat{\Gamma}_{c}\right)}}
$$

In Tables 6.5 (a)-(b), we observe that the spectral approach converges faster than the $h$ discretization. Numerical quadratures are computed as described in [18, Section 3.3].

Table 6.5: Helmholtz convergence when using an overkill $\varphi^{o k}$ with $N_{o k}$ dofs taking as right-hand side $g=\exp (\imath x)$. Notice we slightly abuse notation as this overkill changes from the spectral discretization to the $h$-discretization.

(a) Results for wavenumber $k=1$ using $N_{o k}=Q=97$.

\begin{tabular}{|c|cc|cc|}
\hline & \multicolumn{2}{|c|}{ SPECTRAL DISCRETIZATION } & \multicolumn{2}{c|}{$h$-DISCRETIZATION } \\
\hline $\mathrm{N}$ & $\left\|\varphi^{o k}-\varphi_{N}\right\|_{\widetilde{H}-1 / 2}$ & rel. error & $\left\|\varphi^{o k}-\varphi_{N}\right\|_{\widetilde{H}-1 / 2}$ & rel. error \\
\hline 4 & $1.76 \mathrm{e}-02$ & $1.08 \mathrm{e}-02$ & $1.53 \mathrm{e}+00$ & $3.36 \mathrm{e}-01$ \\
7 & $1.94 \mathrm{e}-06$ & $1.91 \mathrm{e}-06$ & $1.04 \mathrm{e}+00$ & $2.29 \mathrm{e}-01$ \\
13 & $3.21 \mathrm{e}-13$ & $1.97 \mathrm{e}-13$ & $7.25 \mathrm{e}-01$ & $1.59 \mathrm{e}-01$ \\
25 & $7.13 \mathrm{e}-14$ & $4.37 \mathrm{e}-14$ & $5.00 \mathrm{e}-01$ & $1.10 \mathrm{e}-01$ \\
49 & $3.33 \mathrm{e}-14$ & $2.04 \mathrm{e}-14$ & $3.34 \mathrm{e}-01$ & $7.35 \mathrm{e}-02$ \\
\hline
\end{tabular}

(b) Results for wavenumber $k=20$ using $N_{o k}=Q=97$.

\begin{tabular}{|c|cc|cc|}
\hline & \multicolumn{2}{|c|}{ SPECTRAL DISCRETIZATION } & \multicolumn{2}{c|}{$h$-DISCRETIZATION } \\
\hline $\mathrm{N}$ & $\left\|\varphi^{o k}-\varphi_{N}\right\|_{\widetilde{H}^{-1 / 2}}$ & rel. error & $\left\|\varphi^{o k}-\varphi_{N}\right\|_{\widetilde{H}^{-1 / 2}}$ & rel. error \\
\hline 4 & $3.83 \mathrm{e}-00$ & $2.54 \mathrm{e}-01$ & $3.97 \mathrm{e}+01$ & $5.46 \mathrm{e}-01$ \\
7 & $9.74 \mathrm{e}-01$ & $6.48 \mathrm{e}-02$ & $1.90 \mathrm{e}+01$ & $2.61 \mathrm{e}-01$ \\
13 & $9.41 \mathrm{e}-02$ & $6.26 \mathrm{e}-03$ & $1.04 \mathrm{e}+01$ & $1.43 \mathrm{e}-01$ \\
25 & $3.56 \mathrm{e}-04$ & $2.38 \mathrm{e}-05$ & $5.50 \mathrm{e}+00$ & $7.57 \mathrm{e}-02$ \\
49 & $7.84 \mathrm{e}-11$ & $5.22 \mathrm{e}-12$ & $2.80 \mathrm{e}+00$ & $3.85 \mathrm{e}-02$ \\
\hline
\end{tabular}

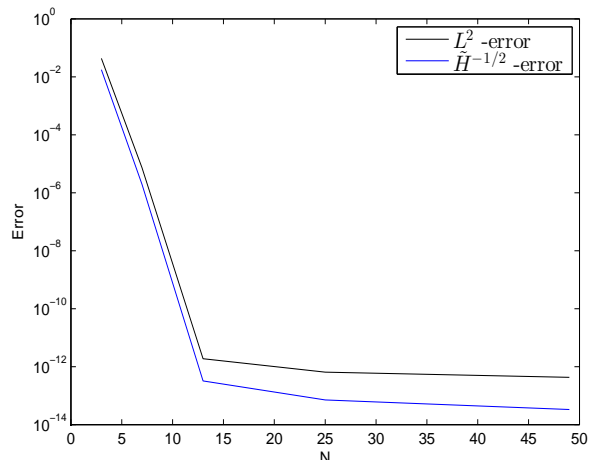

(a) $k=1$

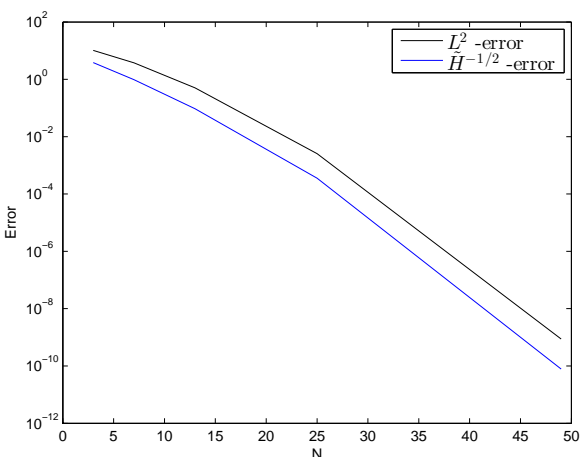

(b) $k=20$

Fig. 6.3. Convergence for wavenumbers $k=1,20$ with right-hand side $g=\exp (\imath x)$. 


\section{Concluding Remarks}

We have introduced a fast convergent method to solve logarithmic singular equations over a segment or smooth Jordan curve using weighted polynomials, both through detailed analysis and numerical experiments. As predicted, convergence rates theoretically obtained, for standard Sobolev spaces, are retrieved in our numerical results for purely logarithmic singular kernels. The strength of the method lies in its fast convergence and simple implementation. These two features become a critical issue when dealing with large numbers of simulations. Moreover, we would like to point out that the method can be directly extended to interface or fracture problems as long as the underlying operator is elliptic. For coercive operators such as Helmholtz one, the method can still be applied. However, the oscillatory nature of the kernel requires more adequate quadrature rules but our preliminary results are promising. Finally, future work seeks to extend these ideas to three-dimensional screens.

\section{A. Technical Lemmas}

Lemma A.1. The space $W$ is continuously imbedded in $\widetilde{H}^{-1 / 2}\left(\Gamma_{c}\right)$.

Proof. This can be obtained from the dense embedding of $L^{2}\left(\Gamma_{c}\right)$ into $\widetilde{H}^{-1 / 2}\left(\Gamma_{c}\right)$ and from Proposition 4.2, which is easily extended to $\Gamma_{c}$ by the coordinate change described in (5.2).

Lemma A.2. Let $\varphi_{R} \in \widetilde{H}^{s-3 / 2}\left(\Gamma_{c}\right)$ for $\frac{5}{2}<s<\frac{7}{2}$. Then, the term $\varphi_{R}^{*}:=\rho^{1 / 2} \varphi_{R}$ belongs to $W$ and it holds

$$
\left\|\varphi_{R}^{*}\right\|_{W} \lesssim\left\|\varphi_{R}\right\|_{\widetilde{H}^{s-3 / 2}\left(\Gamma_{c}\right)} \cdot
$$

Proof. We carry out the analysis for $\hat{\Gamma}_{c}$ without loss of generality. Again, $\rho(x)=w^{2}(x)=$ $1-x^{2}$. First, show that $\varphi_{R}^{*} \in L_{1 / w}^{2}$. By regularity of $\varphi_{R}$ and boundedness of the square-root factor, it holds

$$
\left\|\varphi_{R}^{*}\right\|_{L_{1 / w}^{2}}^{2}=\int_{-1}^{1} \frac{\left|\varphi_{R}^{*}(x)\right|^{2}}{\sqrt{1-x^{2}}} d x=\int_{-1}^{1} \sqrt{1-x^{2}}\left|\varphi_{R}(x)\right|^{2} d x \lesssim\left\|\varphi_{R}\right\|_{\widetilde{H}^{s-3 / 2}\left(\hat{\Gamma}_{c}\right)}^{2} .
$$

Now, we have to prove $\left(\varphi_{R}^{*}\right)^{\prime} \in L_{w}^{2}$. Leibniz's rule provides

$$
\left(\varphi_{R}^{*}\right)^{\prime}(x)=\varphi_{R}^{\prime}(x) \sqrt{1-x^{2}}-\frac{x}{\sqrt{1-x^{2}}} \varphi_{R}(x) .
$$

The first term yields

$$
\int_{-1}^{1}\left|\varphi_{R}^{\prime}(x) \sqrt{1-x^{2}}\right|^{2} \sqrt{1-x^{2}} d x \lesssim \int_{-1}^{1}\left|\varphi_{R}^{\prime}(x)\right|^{2} d x \lesssim\left\|\varphi_{R}\right\|_{\widetilde{H}^{s-3 / 2}\left(\hat{\Gamma}_{c}\right)}^{2},
$$

by regularity of $\varphi_{R}$. The second term gives rise to the integral:

$$
\begin{aligned}
\int_{-1}^{1}\left|\frac{x \varphi_{R}(x)}{\sqrt{1-x^{2}}}\right|^{2} \sqrt{1-x^{2}} d x & =\int_{-1}^{1} \frac{x^{2}}{\sqrt{1-x^{2}}}\left|\varphi_{R}(x)\right|^{2} d x \\
& \leq \int_{-1}^{1} \frac{x^{2} d x}{\sqrt{1-x^{2}}}\left\|\varphi_{R}\right\|_{L^{\infty}\left(\hat{\Gamma}_{c}\right)}^{2} \lesssim\left\|\varphi_{R}\right\|_{\widetilde{H}^{s-3 / 2}\left(\hat{\Gamma}_{c}\right)}^{2} \int_{-1}^{1} \frac{x^{2} d x}{\sqrt{1-x^{2}}},
\end{aligned}
$$


due to the embedding $\widetilde{H}^{s-3 / 2}\left(\hat{\Gamma}_{c}\right) \hookrightarrow \mathcal{C}^{0}\left(\overline{\hat{\Gamma}_{c}}\right)$. By using the variable change $x=\cos \theta$, we obtain

$$
\int_{-1}^{1} \frac{x^{2} d x}{\sqrt{1-x^{2}}}=\int_{0}^{\pi} \cos ^{2} \theta d \theta=\frac{\pi}{2}
$$

which concludes the proof.

Lemma A.3. Let $\psi$ be defined by (5.16). Then, $\psi^{*}:=\rho^{1 / 2} \psi$ belongs to $W$ and

$$
\left\|\psi^{*}\right\|_{W} \lesssim \sum_{ \pm}\left(\left|c_{1 \pm}^{\prime}\right|+\left|c_{2 \pm}^{\prime}\right|\right) .
$$

Proof. As before, we carry out the analysis on $\hat{\Gamma}_{c}$. From (5.16), we clearly have

$$
\psi^{*}=\sum_{ \pm}\left(c_{1 \pm}^{\prime}\left(\eta_{ \pm}-1\right)+c_{2 \pm}^{\prime}\left(\eta_{ \pm}-1\right) \rho\right) .
$$

Since $\rho$ is a polynomial, we see that $\psi^{*}$ and its derivative are uniformly bounded, this implies the conclusion because $w^{-1}$ is integrable.

Lemma A.4. Let $v \in L^{2}\left(\Gamma_{c}\right)$ and $N \in \mathbb{N}$. For all $v_{N} \in \mathbb{Q}_{N}\left(\Gamma_{c}\right)$, define $v_{N}^{*}:=w v_{N} \in L_{1 / w}^{2}$, where $L_{1 / w}^{2}$ is now defined over $\Gamma_{c}$. Then it holds

$$
\left\|v-v_{N}\right\|_{\widetilde{H}^{-1 / 2}\left(\Gamma_{c}\right)} \lesssim\left\|\rho^{1 / 2} v-v_{N}^{*}\right\|_{L_{1 / w}^{2}},
$$

where $L_{1 / w}^{2}$ is now defined over $\Gamma_{c}$.

Proof. By definition,

$$
\left\|v-v_{N}\right\|_{\widetilde{H}^{-1 / 2}\left(\Gamma_{c}\right)}=\sup _{h \in H^{1 / 2}\left(\Gamma_{c}\right)} \frac{\left\langle v-v_{N}, h\right\rangle_{H^{1 / 2}\left(\Gamma_{c}\right)}}{\|h\|_{H^{1 / 2}\left(\Gamma_{c}\right)}} .
$$

Clearly, $v h \in L^{1}\left(\Gamma_{c}\right)$ since it is the product of square-integrable functions. We now show that the product $v_{N} h$ also lies in $L^{1}\left(\Gamma_{c}\right)$. Since $v_{N}$ can be written at an endpoint as $v_{N}(\zeta)=\zeta^{-1 / 2} P(\zeta)$ where $P(\cdot)$ is a polynomial, $v_{N}$ is $L^{p}$-integrable for $p<2$ as locally it holds, for $\epsilon>0$,

$$
\int_{0}^{\epsilon}|\zeta|^{-p / 2} d \zeta<\infty \quad \text { for } \quad-\frac{p}{2}+1>0 .
$$

On the other hand, $H^{1 / 2}\left(\Gamma_{c}\right) \hookrightarrow L^{q}\left(\Gamma_{c}\right)$ for all $q \geq 1$. Hence, $v_{N} h \in L^{1}\left(\Gamma_{c}\right)$ and the duality product can be written as the integral:

$$
\begin{aligned}
\left\langle v-v_{N}, h\right\rangle_{H^{1 / 2}\left(\Gamma_{c}\right)} & =\int_{\Gamma_{c}}\left(v-v_{N}\right) h d x=\int_{\Gamma_{c}} \rho^{1 / 4}\left(v-v_{N}\right) \rho^{-1 / 4} h d x \\
& \leq\left\|\rho^{1 / 4}\left(v-v_{N}\right)\right\|_{L^{2}\left(\Gamma_{c}\right)}\left\|\rho^{-1 / 4} h\right\|_{L^{2}\left(\Gamma_{c}\right)} \lesssim\left\|\rho^{1 / 2} v-v_{N}^{*}\right\|_{L_{1 / w}^{2}}\|h\|_{H^{1 / 2}\left(\Gamma_{c}\right)},
\end{aligned}
$$

where the first inequality results from applying Cauchy-Schwarz while the last one by Lemma 2.1 . 
Lemma A.5. Let $m+\frac{3}{2}<s<m+\frac{5}{2}$. If $\varphi_{R} \in \widetilde{H}^{s-\frac{3}{2}}\left(\Gamma_{c}\right)$, then $\varphi_{R}^{*}:=\rho^{1 / 2} \varphi_{R} \in W^{m}$ and there exists a constant independent of $m$ such that

$$
\left\|\varphi_{R}^{*}\right\|_{W^{m}} \lesssim m 2^{m}\left\|\varphi_{R}\right\|_{\widetilde{H}^{s-\frac{3}{2}}\left(\Gamma_{c}\right)} .
$$

Proof. We carry out the proof on $\hat{\Gamma}_{c}$. By definition, one must show that the $L_{1 / w^{2}}$-norm of $\mathrm{D}^{l}\left(\rho^{1 / 2} \varphi_{R}\right)$ is bounded for all $l=0, \ldots, m$. Again, we exchange $\rho^{1 / 2}$ by $w$ and change coordinates $x$ by $\cos \theta$ to obtain

$$
\left\|\mathrm{D}^{l}\left(w \varphi_{R}\right)\right\|_{1 / w}^{2}=\int_{-1}^{1}\left|\mathrm{D}^{l}\left(w \varphi_{R}\right)\right|^{2} w^{-1} d x=\int_{0}^{\pi}\left|\left(\check{\varphi}_{R}(\theta) \sin \theta\right)^{(l)}\right|^{2} d \theta,
$$

by the mapping $\mathrm{D} \mapsto \mathrm{D}$. On the other hand, it holds

$$
\begin{aligned}
& \left|\left(\check{\varphi}_{R}(\theta) \sin \theta\right)^{(l)}\right|^{2}=\left|\sum_{k=0}^{l}\left(\begin{array}{l}
l \\
k
\end{array}\right) \check{\varphi}_{R}^{(k)}(\theta) \frac{d^{l-k}}{d \theta^{l-k}} \sin \theta\right|^{2} \\
\leq & l \sum_{k=0}^{l}\left(\begin{array}{l}
l \\
k
\end{array}\right)^{2}\left|\check{\varphi}_{R}^{(k)}(\theta)\right|^{2}\left|\frac{d^{l-k}}{d \theta^{l-k}} \sin \theta\right|^{2} .
\end{aligned}
$$

For $l<m$, this leads to

$$
\left|\left(\check{\varphi}_{R} \sin \theta\right)^{(l)}\right|^{2} \leq l \sum_{k=0}^{l}\left(\begin{array}{l}
l \\
k
\end{array}\right)^{2}\left|\check{\varphi}_{R}^{(k)}(\theta)\right|^{2}
$$

Using this in the integral (A.10) above yields

$$
\begin{aligned}
\int_{0}^{\pi}\left|\left(\check{\varphi}_{R}(\theta) \sin \theta\right)^{(l)}\right|^{2} d \theta & \leq l \sum_{k=0}^{l}\left(\begin{array}{l}
l \\
k
\end{array}\right)^{2} \int_{0}^{\pi}\left|\check{\varphi}_{R}^{(k)}(\theta)\right|^{2} d \theta \leq l \sum_{k=0}^{l}\left(\begin{array}{l}
l \\
k
\end{array}\right)^{2} \int_{-1}^{1}\left|\varphi_{R}^{(k)}\right|^{2} w^{-1} d x \\
& \leq \pi l \sum_{k=0}^{l}\left(\begin{array}{l}
l \\
k
\end{array}\right)^{2}\left\|\varphi_{R}^{(k)}\right\|_{L^{\infty}\left(\hat{\Gamma}_{c}\right)}^{2} \leq \pi l 2^{2 l} \max _{k=0, \ldots, l}\left\|\varphi_{R}^{(k)}\right\|_{L^{\infty}\left(\hat{\Gamma}_{c}\right)}^{2}, \quad(\mathrm{~A}
\end{aligned}
$$

since $\left(\begin{array}{l}l \\ k\end{array}\right) \leq 2^{l}$ and $\int_{-1}^{1} w^{-1} d x=\pi$. Due to the continuous embedding of $H^{1}\left(\hat{\Gamma}_{c}\right)$ into $\mathcal{C}^{0}((-1,1))$, we deduce for all $k \leq l$ that

$$
\left\|\varphi_{R}^{(k)}\right\|_{L^{\infty}\left(\hat{\Gamma}_{c}\right)} \lesssim\left\|\varphi_{R}^{(k)}\right\|_{H^{1}\left(\hat{\Gamma}_{c}\right)} \lesssim\left\|\varphi_{R}\right\|_{H^{k+1}\left(\hat{\Gamma}_{c}\right)},
$$

for a constant independent of $k$. Since $k+1 \leq m<s-\frac{3}{2}$, we deduce that

$$
\left\|\varphi_{R}^{(k)}\right\|_{L^{\infty}\left(\hat{\Gamma}_{c}\right)} \lesssim\left\|\varphi_{R}\right\|_{H^{s-\frac{3}{2}}\left(\hat{\Gamma}_{c}\right)} .
$$

This last estimate reveals that

$$
\int_{0}^{\pi}\left|\left(\check{\varphi}_{R}(\theta) \sin \theta\right)^{(l)}\right|^{2} d \theta \lesssim \pi l 2^{2 l}\left\|\varphi_{R}\right\|_{H^{s-\frac{3}{2}}\left(\hat{\Gamma}_{c}\right)}^{2} .
$$

For $l=m$, we distinguish between $k<m$ and $k=m$ to obtain

$$
\left|\left(\check{\varphi}_{R}(\theta) \sin \theta\right)^{(m)}\right|^{2} \leq m \sum_{k=0}^{m-1}\left(\begin{array}{c}
m \\
k
\end{array}\right)^{2}\left|\check{\varphi}_{R}^{(k)}(\theta)\right|^{2}+m \sin ^{2} \theta\left|\check{\varphi}_{R}^{(m)}(\theta)\right|^{2} .
$$


Introducing the above in the integral (A.10) gives

$$
\begin{aligned}
\int_{0}^{\pi}\left|\left(\check{\varphi}_{R}(\theta) \sin \theta\right)^{(m)}\right|^{2} d \theta & \lesssim \pi m 2^{2 m}\left\|\varphi_{R}\right\|_{H^{s-\frac{3}{2}}\left(\hat{\Gamma}_{c}\right)}^{2}+m \int_{0}^{\pi}\left|\check{\varphi}_{R}^{(m)}(\theta)\right|^{2} \sin ^{2} \theta d \theta \\
& \lesssim \pi m 2^{2 m}\left\|\varphi_{R}\right\|_{H^{s-\frac{3}{2}}\left(\hat{\Gamma}_{c}\right)}^{2}+m \int_{-1}^{1}\left|\varphi_{R}^{(m)}\right|^{2} w d x
\end{aligned}
$$

This shows that

$$
\int_{0}^{\pi}\left|\left(\check{\varphi}_{R}(\theta) \sin \theta\right)^{(m)}\right|^{2} d \theta \lesssim\left(\pi m 2^{2 m}+m\right)\left\|\varphi_{R}\right\|_{H^{s-\frac{3}{2}}\left(\hat{\Gamma}_{c}\right)}^{2}
$$

because $m<s-\frac{3}{2}$. Lastly, the two estimates (A.14) and (A.16) imply that

$$
\left\|\varphi_{R}^{*}\right\|_{W^{m}}^{2} \lesssim\left(\pi m 2^{2 m}+m\right) m\left\|\varphi_{R}\right\|_{\widetilde{H}^{s-\frac{3}{2}}\left(\hat{\Gamma}_{c}\right)}^{2},
$$

which leads to the requested estimate.

Lemma A.6. Let $\psi$ be defined by (5.25). Then, $\psi^{*}=\rho^{1 / 2} \psi$ belongs to $W^{m}$ and there exists a positive constant $c(m)$ depending only on $m$ such that

$$
\left\|\psi^{*}\right\|_{W^{m}} \leq c(m) \sum_{ \pm} \sum_{i=0}^{m}\left|c_{i \pm}^{\prime}\right|
$$

Proof. Since $\psi^{*}=\sum_{ \pm}\left(1-\eta_{ \pm}\right) p_{ \pm}$, we easily show that it belongs to $W^{m}$ by using Leibniz rule and Faa di Bruno's formula. The conclusion follows from the definition of $p_{ \pm}$.

Acknowledgments. The authors would like to thank Prof. Christoph Schwab and José Pinto for their insightful remarks over a draft of the current manuscript. This work was supported by Fondecyt Regular 1171491, Conicyt Anillo ACT1417 and CORFO Engineering 2030 program through Grant OPEN-UC 201603.

\section{References}

[1] R. Adams. Sobolev Spaces. Pure and Applied Mathematics: A Series of Monographs and Textbooks. Academic Press, London, UK, 1975.

[2] K. E. Atkinson and I. H. Sloan. The numerical solution of first-kind logarithmic kernel integral equations on smooth open arcs. Mathematics of Computation, 56 (1991), 119-139.

[3] C. Bernardi and Y. Maday. Spectral methods. In P. Ciarlet and J.-L. Lions, editors, Handbook of Numerical Analysis Vol. V, pages 209-485. North-Holland, Amsterdam, 1997.

[4] M. Bourlard, S. Nicaise, and L. Paquet. An adapted Galerkin method for the resolution of Dirichlet and Neumann problems in a polygonal domain. Mathematical Methods in the Applied Sciences, 12 (1990), 251-265.

[5] O. Bruno and S. Lintner. A generalized Calderón formula for open-arc diffraction problems: theoretical considerations Proceedings of the Royal Society of Edinburgh: Section A Mathematics, 145 (2015), 331-364.

[6] O. Bruno and S. Lintner. Second-kind integral solvers for TE and TM problems of diffraction by open arcs. Radio Science, 47 (2012).

[7] C. Canuto, M. Y. Hussaini, A. Quarteroni, and T. A. Zang. Spectral Methods: Fundamentals in Single Domains. Springer-Verlag, Berlin Heidelberg, 2006. 
[8] M. Costabel and M. Dauge. Crack singularities for general elliptic systems. Mathematische Nachrichten, 235 (2002), 29-49.

[9] M. Costabel, M. Dauge, and S. Nicaise. Corner Singularities and Analytic Regularity for Linear Elliptic Systems. Book in preparation.

[10] R. Estrada and R. P. Kanwal. Singular Integral Equations. Birkhäuser, Boston, USA, 2000.

[11] I. Gradshteyn and I. Ryzhik. Table of Integrals, Series, and Products. Academic Press, London, UK, fifth edition, 1994.

[12] P. Grisvard. Elliptic Problems in Nonsmooth Domains. Pitman, London, UK, 1985.

[13] B. Guo and N. Heuer. The optimal rate of convergence of the $p$-version of the boundary element method in two dimensions. Numerische Mathematik, 98 (2004), 499-538.

[14] A. S. Ilynsky. Integral equations for the problems of waves diffraction on screens. In R. Lazarov and V. Petkov, editors, Integral equations and inverse problems, number 235 in Pitman Research Notes in Mathematics Series, pages 128-137. Longman Scientific \& Technical, Essex, UK, 1991.

[15] V. Domínguez, N. Heuer, and F.-J. Sayas, Hilbert scales and Sobolev spaces defined by associated Legendre functions, Journal of Computational and Applied Mathematics, 235 (2011), 3481-3501.

[16] R. Hiptmair, C. Jerez-Hanckes, and C. Urzúa-Torres, Mesh-independent operator preconditioning for boundary elements on open curves, SIAM Journal on Numerical Analysis, 52 (2014), 22952314.

[17] C. Jerez-Hanckes and J. Nédélec, Explicit variational forms for the inverses of integral logarithmic operators over an interval, SIAM Journal on Mathematical Analysis, 44 (2012), 2666-2694.

[18] C. Jerez-Hanckes, J. Pinto, S. Tournier, Local multiple traces formulation for high-frequency scattering problems, Journal of Computational and Applied Mathematics, 289 (2015), 306-321.

[19] R. Kress and I. H. Sloan. On the numerical solution of a logarithmic integral equation of the first kind for the Helmholtz equation. Numerische Mathematik, 66 (1993), 199-214.

[20] J. L. Lions and E. Magenes. Problèmes aux Limites Non Homogènes, volume 1. Dunod, Paris, France, 1968.

[21] J. Mason and D. Handscomb. Chebyshev Polynomials. Chapman \& Hall/CRC, Boca Raton, Florida, USA, 2003.

[22] W. McLean. Strongly Elliptic Systems and Boundary Integral Equations. Cambridge University Press, New York, USA, 2000.

[23] B. Mercier. An Introducition to the Numerical Analysis of Spectral Methods, volume 318 of Lecture Notes in Physics. Springer-Verlag, Heidelberg, Germany, 1989.

[24] S. Mikhlin and S. Prössdorf. Singular Integral Operators. Akademie-Verlag, Berlin, Germany, 1986.

[25] N. I. Muskhelishvili. Singular Integral Equations. Noordhoff International Publishing, Groningen, 1977.

[26] J.-C. Nédélec. Acoustic and Electromagnetic Equations: Integral Representations for Harmonic Problems, volume 144 of Applied Mathematical Sciences. Springer-Verlag, New York, USA, 2001.

[27] S. Nicaise and A.-M. Sändig. General interface problems I. Mathematical Methods in Applied Sciences, 17 (1994), 395-429.

[28] S. Nicaise and A.-M. Sändig. General interface problems II. Mathematical Methods in Applied Sciences, 17 (1994), 431-450.

[29] J. Saranen and G. Vainikko. Periodic Integral and Pseudodifferential Equations with Numerical Approximation. Springer Monographs in Mathematics. Springer-Verlag, Heildelberg, Germany, 2002 .

[30] S. A. Sauter and C. Schwab. Boundary element methods, volume 39 of Springer Series in Computational Mathematics. Springer-Verlag, Berlin, 2011.

[31] Y. V. Shestopalov, Y. G. Smirnov, and E. V.Chernokozhin. Logarithmic Integral Equations in Electromagnetics. VSP, Utrecht, Netherlands, 2000.

[32] S. Steinbach, Numerical approximation methods for elliptic boundary value problems: Finite and 
boundary elements, Springer, New York, 2008.

[33] E. Stephan. Boundary integral equations for screen problems in $\mathbb{R}^{3}$. Integral Equations Operator Theory, 10 (1987), 236-257.

[34] E. Tadmor. The exponential accuracy of Fourier and Chebyshev differencing methods. SIAM Journal on Numerical Analysis, 23 (1986), 1-10.

[35] L. Tartar. An Introduction to Sobolev Spaces and Interpolation Spaces, volume 3 of Lecture Notes of the Unione Matematica Italiana. Springer, Heildelberg, Germany, 2007. 